TRANSACTIONS OF THE

AMERICAN MATHEMATICAL SOCIETY

Volume 362, Number 8, August 2010, Pages 3911-3946

S 0002-9947(10)05170-6

Article electronically published on March 1, 2010

\title{
REPRESENTATION AND INDEX THEORY FOR TOEPLITZ OPERATORS
}

\author{
G. J. MURPHY
}

\begin{abstract}
We study Toeplitz operators on the Hardy spaces of connected compact abelian groups and of tube-type bounded symmetric domains. A representation theorem for these operators and for classes of abstract Toeplitz elements in $\mathrm{C}^{*}$-algebras is proved. This is used to give a unified treatment to index theory in this setting, and a variety of new index theorems are proved that generalize the Gohberg-Krein theorem for Toeplitz operators on the Hardy space of the unit circle in the plane.
\end{abstract}

\section{INTRODUCTION}

This paper is a study of various classes of Toeplitz operators acting on generalized Hardy Hilbert spaces, principally those associated to abelian connected compact groups and to bounded symmetric domains, but also including Hardy spaces obtained through a Hilbert module construction. The theory we study is a generalization of the theory of Toeplitz operators on the Hardy Hilbert space $H^{2}$ of the circle group $\mathbf{T}$ in the complex plane $\mathbf{C}$.

There are two important aspects to the study of Toeplitz operators: their spectral theory and their index theory. In an earlier paper [15] we have made a detailed study of spectral theory in a very general Hardy space framework. Here we concentrate on index theory.

If $f$ is a continuous function on $\mathbf{T}$ and $T_{f}$ is the corresponding Toeplitz operator on $H^{2}$, then the well-known theorem of I. Gohberg and M. Krein 12, Theorem 3.5.15] asserts that $T_{f}$ is a Fredholm operator if, and only if, $f$ never vanishes and, in this case,

$$
\operatorname{index}\left(T_{f}\right)=-\operatorname{wn}(f),
$$

where index $\left(T_{f}\right)$ is the Fredholm index of $T_{f}$ and $w n(f)$ is the winding number of $f$ around the origin. One of our major objectives in this paper is to obtain a far-reaching generalization of this result.

The Gohberg-Krein index theorem has already inspired many significant generalizations, including the Atiyah-Singer index theorem and, indeed, much of the rest of index theory, as is well known. Since there already exist a number of index theorems for Toeplitz operators, we should explain what is new about our approach. Firstly, our emphasis here is not on obtaining an index theorem per se - such theorems can be very abstract, involving the elaborate machinery of $K$-theory and Kasparov's $K K$-theory, Atiyah-Singer index theory and many other ingredients

Received by the editors January 23, 2006.

2000 Mathematics Subject Classification. Primary 47B35, 46L05, 46L08, 43A17.

(C)2010 American Mathematical Society Reverts to public domain 28 years from publication 
(see the formidable index theorem of H. Upmeier on Toeplitz operators on bounded symmetric domains [25], for example). Our objective here is more specific: to develop an index theorem that is as much like the classical Gohberg-Krein theorem as possible. Thus, we want to involve something like a Fredholm operator index and some kind of topological index function corresponding to the winding number in the case of the circle. Secondly, and very importantly, we want to give a treatment that puts the index theorems for the various classes of Toeplitz operators we consider into a unified general framework (this has not been done before). Thirdly, we shall emphasize "naturalness" of our index results. Our operator index will be defined in terms of a trace, and we shall see in examples that there is often a natural choice for the trace to obtain the appropriate operator index. Again, for the topological index, we shall usually define this in terms of a derivation that is naturally distinguished in some sense in the algebra of symbols for the Toeplitz operators.

It is clear from earlier studies of index theory in the setting of generalized Hardy spaces that one is going to have to modify both sides of equation (1) in order to extend the result appropriately. The usual integer-valued Fredholm index has been replaced by the real-valued index of M. Breuer [5, 6] in some cases. From our point of view this is not entirely satisfactory, since it requires a representation of the Toeplitz operators on a different Hilbert space (from its Hardy space domain) in order even to define the Breuer-Fredholm index of the operator [7, 16. In our approach, the index theorem can be given a completely intrinsic formulation. Nevertheless, we shall develop a representation theory also, but this will involve no ad hoc elements and its use in the index theorem is to provide a unifying framework that does not substitute for, but rather facilitates, an intrinsic formulation.

In addition to obtaining a suitable analytic (Fredholm-type) index for operators, there is also the problem of obtaining a suitable topological index $\omega(f)$ for functions $f$ that generalizes the winding number. Although many generalizations of the winding number have been found in various different settings - such as the average winding number in the case of almost periodic functions - nevertheless the definition of the analogue of the winding number in each case has been made on a somewhat ad hoc basis. In this paper we present a systematic approach in terms of naturally distinguished derivations and traces on the algebra of symbol functions.

Our index theorems are derived within a unifying framework provided by a representation theorem we prove that shows that the classes of Toeplitz operators on Hardy spaces of abelian connected compact groups and Hardy spaces of tube-type bounded symmetric domains can be represented as Toeplitz operators on standard Hilbert modules. This latter class of Toeplitz operators has operator matrices with constant diagonals, in analogy with the classical Toeplitz operators on $H^{2}$. We initiate the study of these operators and prove an index theorem for them.

Given the variety of classes of Toeplitz operators that have been considered in the literature, it is desirable to develop an abstract theory that provides a general framework for their analysis. We do this here, for very large classes of Toeplitz operators, by defining Toeplitz elements in a $\mathrm{C}^{*}$-algebra relative to a fixed nonunitary isometry. We show that, subject to mild restrictions on the isometry, our representation theorem realizes these abstractly-defined Toeplitz elements concretely as Toeplitz operators on a standard Hilbert module. We also prove various useful results for the abstract Toeplitz elements that can be applied in particular cases. 
We outline now how the paper is organized. In Section 2 we develop an approach to topological index theory, where the index on the invertible elements of a $\mathrm{C}^{*}$ algebra is defined in terms of a densely-defined derivation in the algebra. This fits in very naturally with how index theory works for symbols of Toeplitz operators. In Section 3 we introduce and develop the theory of Toeplitz operators on Hilbert modules and prove our first index theorem in this setting. In the following section, Section 4 , we define an abstract theory of Toeplitz elements in $\mathrm{C}^{*}$-algebras that gives a unified framework for the various classes of Toeplitz operators considered in this paper. We show that Toeplitz elements can be concretely represented as Toeplitz operators on Hilbert modules. In Section 5 we illustrate the scope of our theory by showing that large classes of examples fit within its framework. Toeplitz operators on (most) abelian connected compact groups and on tube-type bounded symmetric domains are examples of Toeplitz elements in $\mathrm{C}^{*}$-algebras. They therefore have a representation on Hilbert modules; an advantage of this representation for these classes of operators is that it provides a mechanism for constructing a trace on the relevant Toeplitz algebra (the $\mathrm{C}^{*}$-algebra generated by the class of Toeplitz operators that one is considering) that defines the generalized Fredholm index one needs to prove an intrinsic index theorem for these examples. The final two sections, Sections 6 and 7, develop the index theory for Toeplitz operators on the Hardy spaces of compact groups and symmetric domains, respectively.

We fix some notation now that we will use throughout the paper: If $H$ is a Hilbert space, we denote the $\mathrm{C}^{*}$-algebra of norm-bounded linear operators on $H$ by $\mathcal{B}(H)$ and the ideal of compact operators on $H$ by $\mathcal{K}(H)$. If $\left(f_{i}\right)_{i \in I}$ is some orthonormal basis for $H$, we denote by $f_{i j} \in \mathcal{K}(H)$ the rank-one operator $f \mapsto\left\langle f_{j} \mid f\right\rangle f_{i}$.

Note that we are writing the inner product on Hilbert spaces so that it is linear in the second variable and conjugate-linear in the first. We do this to maintain consistency with the definition of a Hilbert module, where the inner product is usually assumed to be linear in the second variable; see [10. Hilbert modules will play a central role in this paper.

\section{TOPOLOGICAL INDEX THEORY}

As indicated in the Introduction, we shall be interested in proving a number of index theorems in this paper. For this purpose we'll need the concept of a topological index. Our aim here is to define this concept and prove some existence theorems.

The prototype of a topological index is the winding number function

$$
\text { wn }: \operatorname{Inv} C(\mathbf{T}) \rightarrow \mathbf{Z}, \quad f \mapsto \operatorname{wn}(f),
$$

on the invertible (nonvanishing) continuous functions on the circle. For a continuously differentiable function $f$ in $\operatorname{Inv} C(\mathbf{T})$ (meaning that the function $t \mapsto f\left(e^{i t}\right)$ is continuously differentiable everywhere on $\mathbf{R}$ ), we have the familiar formula

$$
\operatorname{wn}(f)=\frac{1}{2 i \pi} \int_{0}^{2 \pi} \frac{1}{f\left(e^{i t}\right)} \frac{\mathrm{d} f\left(e^{i t}\right)}{\mathrm{d} t} \mathrm{~d} t .
$$

We rewrite this in an equivalent way that permits generalization. For each continuously differentiable function $f \in C(\mathbf{T})$, write $D f$ for the continuous function on $\mathbf{T}$ associated to the periodic function

$$
\mathbf{R} \rightarrow \mathbf{C}, \quad t \mapsto-i \frac{\mathrm{d} f\left(e^{i t}\right)}{\mathrm{d} t} .
$$


Then

$$
\operatorname{wn}(f)=\int f^{-1} D f \mathrm{~d} m,
$$

where $m$ is the normalized Haar measure on $\mathbf{T}$. Of course, $D$ is a derivation, namely, the Dirac operator of the circle.

We shall usually want to consider only those topological indices that are defined in this way: that is, in association with a suitable derivation.

Let us also note that it is desirable to treat Toeplitz operators with symbols in noncommutative algebras as well as in commutative ones, since Toeplitz operators on Hilbert modules have symbols in a noncommutative algebra. This requires noncommutative integration theory; that is, we must consider traces as well as integration against measures.

Let $\mathcal{A}$ be a unital $\mathrm{C}^{*}$-algebra. A topological index on $\mathcal{A}$ is a locally constant function $\omega: \operatorname{Inv} \mathcal{A} \rightarrow \mathbf{C}$ such that

$$
\omega(a b)=\omega(a)+\omega(b),
$$

for all $a, b \in \operatorname{Inv} \mathcal{A}$.

Of course, local constancy is equivalent to continuity, where $\mathbf{C}$ is endowed with the discrete topology.

Clearly, $\omega(1)=0$. Since, for all $a \in \mathcal{A}$, the function

$$
[0,1] \mapsto \mathbf{C}, \quad t \mapsto \omega\left(e^{t a}\right),
$$

is locally constant and has connected domain, it must be a constant function. Hence, $\omega\left(e^{a}\right)=\omega(1)=0$. It follows that if $b \in \mathcal{A}$ and $\|1-b\|<1$, then $\omega(b)=0$ (since $b=e^{a}$, for some element $a \in \mathcal{A}$ ).

We say that a unital subalgebra $\mathcal{A}_{0}$ of a unital $\mathrm{C}^{*}$-algebra $\mathcal{A}$ is inverse closed in $\mathcal{A}$ if whenever $a \in \mathcal{A}_{0}$ is invertible in $\mathcal{A}$, its inverse belongs to $\mathcal{A}_{0}$. In this case, Inv $\mathcal{A}_{0}=\mathcal{A}_{0} \cap \operatorname{Inv} \mathcal{A}$, and conversely. If $\mathcal{A}_{0}$ is dense and inverse closed in $\mathcal{A}$, then $\operatorname{Inv} \mathcal{A}_{0}$ is dense in $\operatorname{Inv} \mathcal{A}$.

We shall need the following elementary result.

Lemma 2.1. If $\mathcal{A}_{0}$ is an inverse-closed dense unital subalgebra of a unital $C^{*}$ algebra $\mathcal{A}$ and $\omega: \operatorname{Inv} \mathcal{A}_{0} \rightarrow \mathbf{C}$ is a function such that equation (2) holds and $\omega(a)=0$ whenever $a \in \mathcal{A}_{0}$ and $\|1-a\|<1$, then $\omega$ extends uniquely to a topological index $\omega: \operatorname{Inv} \mathcal{A} \rightarrow \mathbf{C}$.

Proof. Suppose that $a, b \in \operatorname{Inv} \mathcal{A}_{0}$ and $\|b-a\|<\left\|a^{-1}\right\|^{-1}$. Then $\left\|b a^{-1}-1\right\|<1$ and therefore, since $b a^{-1} \in \mathcal{A}_{0}, \omega\left(b a^{-1}\right)=0$. Hence, $\omega(b)=\omega\left(b a^{-1}\right)+\omega(a)=\omega(a)$. Thus, $\omega$ is locally constant on $\operatorname{Inv} \mathcal{A}_{0}$.

Now suppose that $a$ and $b$ are elements of $\operatorname{Inv} \mathcal{A}$ such that $\|b-a\|<\left\|a^{-1}\right\|^{-1}$. Then there exist sequences $\left(a_{n}\right)$ and $\left(b_{n}\right)$ in Inv $\mathcal{A}_{0}$ converging to $a$ and $b$, respectively, and, for any such sequences there exists a positive integer $N$ such that $\left\|b_{n}-a_{n}\right\|<\left\|a_{n}{ }^{-1}\right\|^{-1}$, for all $n \geq N$. Hence, $\omega\left(a_{n}\right)=\omega\left(b_{n}\right)$, for all $n \geq N$. This shows that for any sequence $\left(a_{n}\right)$ in $\operatorname{Inv} \mathcal{A}_{0}$ converging to $a$, the sequence $\left(\omega\left(a_{n}\right)\right)$ is eventually constant and the value it attains is independent of the choice of sequence $\left(a_{n}\right)$. We set $\omega(a)=\lim _{n} \omega\left(a_{n}\right)$. We also see now that if $\|b-a\|<\left\|a^{-1}\right\|^{-1}$, then since $\omega\left(a_{n}\right)=\omega\left(b_{n}\right)$ for $n \geq N$, we have $\omega(a)=\omega(b)$. Hence, $a \mapsto \omega(a)$ is a locally constant function on $\operatorname{Inv} \mathcal{A}$ extending uniquely the function $\omega: \operatorname{Inv} \mathcal{A}_{0} \rightarrow \mathbf{C}$. Since equation (2) holds for arbitrary elements of Inv $\mathcal{A}_{0}$, it also holds for arbitrary elements of $\operatorname{Inv} \mathcal{A}$, by density of $\operatorname{Inv} \mathcal{A}_{0}$ in $\operatorname{Inv} \mathcal{A}$ and continuity of $\omega$ relative to the discrete topology on $\mathbf{C}$. 
A derivation defined in a unital $\mathrm{C}^{*}$-algebra $\mathcal{A}$ is a linear map $D: \mathcal{A}_{0} \rightarrow \mathcal{A}$ from a unital subalgebra $\mathcal{A}_{0}$ of $\mathcal{A}$ to $\mathcal{A}$ such that the Leibnitz condition holds:

$$
D(a b)=(D a) b+a D b \quad\left(a, b \in \mathcal{A}_{0}\right) .
$$

Clearly, $D 1=0$ and, if $a, a^{-1} \in \mathcal{A}_{0}, D\left(a^{-1}\right)=-a^{-1}(D a) a^{-1}$.

We say a derivation $D$ is skew-adjoint if its domain $\mathcal{A}_{0}$ is selfadjoint and $D\left(a^{*}\right)=$ $-(D a)^{*}$, for all $a \in \mathcal{A}_{0}$.

Theorem 2.2. Let $D: \mathcal{A}_{0} \rightarrow \mathcal{A}$ be a derivation in a unital $C^{*}$-algebra $\mathcal{A}$ such that its domain $\mathcal{A}_{0}$ is an inverse-closed dense subalgebra of $\mathcal{A}$. Let $\tau$ be a tracial state on $\mathcal{A}$ such that $\tau(D a)=0$, for all $a \in \mathcal{A}_{0}$. Then there is a unique topological index $\omega: \operatorname{Inv} \mathcal{A} \rightarrow \mathbf{C}$ such that

$$
\omega(a)=\tau\left(a^{-1} D a\right)
$$

for all $a \in \operatorname{Inv} \mathcal{A}_{0}$.

If $D$ is skew-adjoint, then $\omega$ is real-valued.

Proof. If $a \in \operatorname{Inv} \mathcal{A}_{0}$, set $\omega(a)=\tau\left(a^{-1} D a\right)$. Then, for all $a, b \in \operatorname{Inv} \mathcal{A}_{0}$, we have $\omega(a b)=\tau\left(b^{-1} a^{-1}((D a) b+a D b)\right)=\tau\left(a^{-1}(D a) b b^{-1}\right)+\tau\left(b^{-1} D b\right)=\omega(a)+\omega(b)$.

If $a$ and $b$ are commuting elements in $\mathcal{A}_{0}$, then, for all $n>0$,

$$
\tau\left(b D\left(a^{n}\right)\right)=n \tau\left(b a^{n-1} D a\right) .
$$

We show this by induction on $n$. It clearly holds for $n=1$. Suppose then it holds (for all commuting pairs) for some value $n$ and we'll see it holds also for $n+1$. We have $\tau\left(b D\left(a^{n+1}\right)\right)=\tau\left(b a D\left(a^{n}\right)\right)+\tau\left(b(D a) a^{n}\right)=n \tau\left(b a a^{n-1} D a\right)+\tau\left(b a^{n} D a\right)=$ $(n+1) \tau\left(b a^{n} D a\right)$. It follows that $\tau\left(a^{n} D a\right)=0$, for all $n \geq 0$, since $(n+1) \tau\left(a^{n} D a\right)=$ $\tau\left(D\left(a^{n+1}\right)\right)=0$.

Now suppose $a \in \mathcal{A}_{0}$ and $\|1-a\|<1$. Then $\omega(1-a)=\tau\left((1-a)^{-1} D(1-a)\right)=$ $-\tau\left(\sum_{n=0}^{\infty} a^{n} D a\right)=-\sum_{n=0}^{\infty} \tau\left(a^{n} D a\right)=0$. The existence of the topological index on $\operatorname{Inv} \mathcal{A}$ extending $\omega$ on $\operatorname{Inv} \mathcal{A}_{0}$ now follows from Lemma 2.1. We denote this extension by $\omega$ also.

Suppose now that $D$ is skew-adjoint. If $a$ is an element of $\operatorname{Inv} \mathcal{A}_{0}$, then $\omega\left(a^{*}\right)=$ $\tau\left(\left(a^{*}\right)^{-1} D\left(a^{*}\right)\right)=-\tau\left(a^{-1} D a\right)^{-}=-\omega(a)^{-}$. However, $a^{*} a=e^{b}$, for some element $b \in \mathcal{A}$, so $\omega\left(a^{*} a\right)=0$; that is, $\omega\left(a^{*}\right)+\omega(a)=0$. Hence, $\omega\left(a^{*}\right)=-\omega(a)$. Thus, $\omega(a)^{-}=-\omega\left(a^{*}\right)=\omega(a)$, showing $\omega(a)$ is real-valued in this case, as required.

The condition $\tau(D a)=0$ is not always easy to check directly in examples. The framework discussed in the following example offers an approach that gets around this difficulty. This links the discussion here with a similar circle of ideas that have been considered by other authors (see 21] and the references therein).

Example 2.3. Let $\mathcal{A}$ be a unital $\mathrm{C}^{*}$-algebra and let $\alpha$ be a flow on $A$; that is, $\alpha$ is a homomorphism from the additive group $\mathbf{R}$ to the automorphism group Aut $\mathcal{A}$ of $\mathcal{A}$ that is strongly continuous in the sense that, for all $a \in \mathcal{A}$, the map $\mathbf{R} \rightarrow \mathcal{A}$, $t \mapsto \alpha_{t}(a)$, is continuous. It is well known that the set $\mathcal{A}_{\alpha}$ of all elements $a$ of $\mathcal{A}$ for which $\lim _{t \rightarrow 0}\left(\alpha_{t}(a)-a\right) / t$ exists is a dense inverse-closed $*$-subalgebra of $\mathcal{A}$ and that the map

$$
D_{\alpha}: \mathcal{A}_{0} \rightarrow \mathcal{A}, \quad a \mapsto-i \lim _{t \rightarrow 0}\left(\alpha_{t}(a)-a\right) / t
$$

is a skew-adjoint derivation of $\mathcal{A}\left(i D_{\alpha}\right.$ is, of course, the infinitesimal generator of $\alpha$; however, we shall find it more convenient to work with $D_{\alpha}$, since we can use it to get a real-valued topological index). 
If $\tau$ is a tracial state on $\mathcal{A}$ that is $\alpha$-invariant (that is, $\tau \circ \alpha_{t}=\tau$, for all $t \in \mathbf{R}$ ), then clearly $\tau\left(D_{\alpha} a\right)=0$, for all $a \in \mathcal{A}_{\alpha}$. Hence, by Theorem 2.2, there is a unique topological index $\omega: \operatorname{Inv} \mathcal{A} \rightarrow \mathbf{R}$ such that $\omega(a)=\tau\left(a^{-1} D_{\alpha} a\right)$, for all $a \in \mathcal{A}_{\alpha}$.

We shall call $\omega$ the topological index associated to $\alpha$ and $\tau$.

Recall that an ordered group is an abelian group $\Gamma$ endowed with a linear (total) order relation $\leq$ that is translation-invariant; that is, if $x, y, z \in \Gamma$ and $x \leq y$, then $x+z \leq y+z$. The ordered subgroups of $\mathbf{R}$ provide an uncountable variety of examples. However, the class of ordered groups is much vaster than this, since every torsion-free abelian group admits a total order making it an ordered group. If $G$ is a compact abelian group with discrete Pontryagin dual group $\Gamma$, then $G$ is connected if, and only if, $\Gamma$ admits a total order relation making it an ordered group ( $\Gamma$ may, and often does, admit many such total order relations). For details on ordered groups (and their Hardy spaces that we consider later), see 23.

If $\Gamma$ is an ordered group, we denote by $\Gamma^{+}$the subsemigroup of all elements $x \in \Gamma$ such that $x \geq 0$ and call the elements of $\Gamma^{+}$the positive elements of $\Gamma$.

A homomorphism $\varphi: \Gamma_{1} \rightarrow \Gamma_{2}$ between ordered groups is said to be positive if $\varphi\left(\Gamma_{1}^{+}\right) \subseteq \Gamma_{2}^{+}$. It is an order isomorphism if, in addition, it is bijective.

The case where an ordered group $\Gamma$ is Archimedean is especially noteworthy; this is the case where, for all $x, z \in \Gamma$ with $z>0$, there exists a positive integer $N$ such that $x \leq N z$. Equivalently, $\Gamma$ is order isomorphic to an ordered subgroup of $\mathbf{R}$.

Suppose now that $\Gamma$ admits an order unit; that is, an element $z$ such that for all $x \in \Gamma$, there exists a positive integer $N$ such that $x \leq N z$. Clearly $z$ is necessarily positive.

We note that $\Gamma$ admits an order unit $z$ if it is finitely generated. For in this case we can choose positive generators $x_{1}, \ldots, x_{N}$ and set $z=x_{1}+\cdots+x_{N}$. If $\Gamma$ is an arbitrary ordered subgroup of $\mathbf{R}$, every nonzero positive element of $\Gamma$ is an order unit, by the Archimedean property.

In some sense these remarks indicate that "most" ordered groups admit an order unit. The existence of order units will play a crucial role in the sequel, so it is pertinent to observe that not all ordered groups admit order units. For example, the set $\mathbf{Z}^{\infty}$ of all sequences of integers that have all but finitely many terms equal to zero is an ordered group under pointwise-defined addition and the order relation $\leq$ defined by setting $x<y$ if, at the greatest integer $n$ for which $x_{n} \neq y_{n}, x_{n}<y_{n}$. It is easily verified that $\mathbf{Z}^{\infty}$ has no order unit.

An order ideal in an ordered group $\Gamma$ is a subgroup $I$ of $\Gamma$ such that if $0 \leq x \leq y$ and $x \in \Gamma$ and $y \in I$, then $x \in I$. It is easy to see that for any two order ideals $I$ and $J$ of $\Gamma$, we have either $I \subseteq J$ or $J \subseteq I$. It is well known that $\Gamma$ is Archimedean if, and only if, its only order ideals are the trivial ones, 0 and $\Gamma$.

If $\Gamma$ is an ordered group and $z$ is an order unit for $\Gamma$, then an order ideal $I$ containing $z$ obviously must be equal to $\Gamma$. It follows that there is a unique largest proper order ideal $I$ of $\Gamma$, if $\Gamma$ is nonzero and admits an order unit (namely the union of all the proper order ideals of $\Gamma$ ). Conversely, if $\Gamma$ has a largest proper order ideal $I$, then $\Gamma$ admits an order unit. To see this, observe that the quotient group $\Gamma / I$ is an ordered group for the unique translation-invariant order making the quotient homomorphism $\Gamma \rightarrow \Gamma / I$ a positive map. Clearly, $\Gamma / I$ is Archimedean and therefore if $z$ is a positive element of $\Gamma$ not contained in $I$, then $z+I$ is a nonzero positive element of $\Gamma / I$ and hence $z+I$ is an order unit of $\Gamma / I$. From 
this it follows that $z$ is an order unit of $\Gamma$. For, if $x \in \Gamma$, then there is a positive integer $N$ such that $x+I<N z+I$ and therefore $x<N z$.

We shall see below that the hypothesis on an ordered group that it admit an order unit is one that is totally natural in the context of index theory.

We shall always assume henceforth that ordered groups are nonzero, to avoid trivialities.

Lemma 2.4. If $\Gamma$ is an ordered group, then there exists a nonzero positive homomorphism $\varphi: \Gamma \rightarrow \mathbf{R}$ if, and only if, $\Gamma$ admits an order unit. In this case, $\varphi$ is unique up to multiplication by a positive scalar and, for every positive element $z$ of $\Gamma, \varphi(z)>0$ if, and only if, $z$ is an order unit of $\Gamma$.

Proof. Let $z$ be an order unit of $\Gamma$ and let $I$ be the largest proper ideal of $\Gamma$. The quotient group $\Gamma^{\prime}=\Gamma / I$ is an ordered group and the quotient homomorphism $\pi: \Gamma \rightarrow \Gamma^{\prime}$ a positive map and clearly $\Gamma^{\prime}$ has no nontrivial order ideals; that is, $\Gamma^{\prime}$ is Archimedean. Hence, there exists an injective positive homomorphism $\varphi^{\prime}: \Gamma^{\prime} \rightarrow \mathbf{R}$. Then $\varphi=\varphi^{\prime} \circ \pi$ is a positive homomorphism from $\Gamma$ to $\mathbf{R}$ and is nonzero since $\varphi(z)>0$.

Now suppose $\varphi_{1}: \Gamma \rightarrow \mathbf{R}$ is another nonzero positive homomorphism from $\Gamma$ to R. Then $\Gamma / \operatorname{ker}\left(\varphi_{1}\right)$ is an Archimedean group and therefore $\operatorname{ker}\left(\varphi_{1}\right)$ is a maximal proper ideal of $\Gamma$. Hence, $\operatorname{ker}\left(\varphi_{1}\right)=I$. Since $\varphi_{1}(I)=0$, there exists an injective positive homomorphism $\varphi_{1}^{\prime}: \Gamma^{\prime} \rightarrow \mathbf{R}$ such that $\varphi_{1}=\varphi_{1}^{\prime} \circ \pi$. Now the map

$$
\theta: \varphi^{\prime}\left(\Gamma^{\prime}\right) \rightarrow \varphi_{1}^{\prime}\left(\Gamma^{\prime}\right), \quad \varphi^{\prime}(x) \mapsto \varphi_{1}^{\prime}(x),
$$

is an order isomorphism between ordered subgroups of $\mathbf{R}$ and therefore, by an elementary argument, there is a positive number $c$ such that $\theta(y)=c y$, for all $y \in \varphi^{\prime}\left(\Gamma^{\prime}\right)$. Hence, for all $x \in \Gamma, \varphi_{1}(x)=c \varphi(x)$.

Now suppose conversely that there exists a nonzero positive homomorphism $\varphi: \Gamma \rightarrow \mathbf{R}$. If $I=\operatorname{ker}(\varphi)$ and $z$ is a positive element of $\Gamma$ for which $\varphi(z)>0$, then $z+I$ is a nonzero positive element of the Archimedean ordered group $\Gamma / I$, and is therefore an order unit of $\Gamma / I$. Hence, if $x \in \Gamma$, there exists a positive integer $N$ such that $x+I<N z+I$ and therefore $x<N z$. Hence, $z$ is an order unit of $\Gamma$.

It is useful to recall here the following result, since we shall make use of it in our next theorem and again in the sequel.

Theorem 2.5 (Bohr-Van Kampen [4, 27). Let $G$ be a connected compact group. If $f: G \rightarrow \mathbf{C}$ is a continuous nonvanishing function, then there exists a continuous character $\chi: G \rightarrow \mathbf{T}$ and a continuous function $h: G \rightarrow \mathbf{C}$ such that $f=\chi e^{h}$.

Let $\Gamma$ be an ordered group, so that the dual group $G=\hat{\Gamma}$ is a compact connected group. We denote by $L^{2}(G)$ its Hilbert space of square-integrable functions relative to the normalized Haar measure on $G$. Recall that the family of elements $\left(\varepsilon_{x}\right)_{x \in \Gamma}$ forms an orthonormal basis for $L^{2}(G)$, where $\varepsilon_{x}$ is the continuous function on $G$ given by evaluation at $x, \varepsilon_{x}(g)=g(x)$.

Denote by $\operatorname{TP}(G)$ the dense $*$-subalgebra of $C(G)$ consisting of the trigonometric polynomials; that is, the linear span of the elements $\varepsilon_{x}(x \in \Gamma)$.

We shall say that a derivation $D: \operatorname{TP}(G) \rightarrow C(G)$ is $G$-invariant if $D \alpha_{g}(f)=$ $\alpha_{g} D(f)$, for all $f \in \operatorname{TP}(G)$, where the map

$$
\alpha: G \rightarrow \operatorname{Aut} C(G), \quad g \mapsto \alpha_{g},
$$


is the homomorphism given by left translation:

$$
\alpha_{g}(f)\left(g^{\prime}\right)=f\left(g^{-1} g^{\prime}\right) \quad\left(g, g^{\prime} \in G, f \in C(G)\right) .
$$

Theorem 2.6. Let $\Gamma$ be an ordered group admitting an order unit and let $G$ be its dual group. Then there is a nonzero topological index $\omega: \operatorname{Inv} C(G) \rightarrow \mathbf{R}$, unique up to multiplication by a positive scalar, such that $\omega\left(\varepsilon_{x}\right) \geq 0$, for all $x \in \Gamma^{+}$. Moreover, there is a unique $G$-invariant skew-adjoint derivation $D: \operatorname{TP}(G) \rightarrow C(G)$ such that

$$
\omega(f)=\int f^{-1} D f \mathrm{~d} m \quad(f \in \operatorname{TP}(G) \cap \operatorname{Inv}(G)),
$$

where $m$ is the normalized Haar measure on $G$.

Proof. Let $z$ be an order unit for $\Gamma$ and let $\varphi: \Gamma \rightarrow \mathbf{R}$ be the unique positive homomorphism for which $\varphi(z)=1$. For each number $t$ in $\mathbf{R}$ we get an element $g_{t} \in G$ by setting $g_{t}(x)=e^{i t \varphi(x)}$, for all $x \in \Gamma$. It follows that if $\alpha: G \rightarrow$ Aut $C(G)$ is the homomorphism given by left translation, then the map

$$
\beta: \mathbf{R} \rightarrow \operatorname{Aut} C(G), \quad t \mapsto \beta_{t}=\alpha_{g_{t}-1},
$$

is a homomorphism. This is clearly strongly continuous, since $\beta_{t}\left(\varepsilon_{x}\right)=e^{i \varphi(x) t} \varepsilon_{x}$, which shows that the map $t \mapsto \beta_{t}\left(\varepsilon_{x}\right)$ is continuous, for all $x \in \Gamma$, and since the elements $\varepsilon_{x}$ generate $C(G)$, it follows that the map $t \mapsto \beta_{t}(f)$ is continuous, for all $f \in C(G)$.

Let $D_{\beta}: \mathcal{A}_{\beta} \rightarrow C(G)$ be the skew-adjoint derivation associated to $\beta$. Since $\lim _{t \rightarrow 0}\left(\beta_{t}\left(\varepsilon_{x}\right)-\varepsilon_{x}\right) / t=\varepsilon_{x} \lim _{t \rightarrow 0}\left(e^{i \varphi(x) t}-1\right) / t=i \varphi(x) \varepsilon_{x}$, we have $\operatorname{TP}(G) \subseteq \mathcal{A}_{\beta}$ and $D\left(\varepsilon_{x}\right)=\varphi(x) \varepsilon_{x}$, for all $x \in \Gamma$, where $D$ is the restriction of $D_{\beta}$ to $\operatorname{TP}(G)$ (thus, $D$ is a skew-adjoint derivation). One easily checks that $D$ is $G$-invariant.

If $f \in C(G)$, then $\int \alpha_{g}(f) \mathrm{d} m=\int f \mathrm{~d} m$, by invariance of $m$. Hence, $\int \beta_{t}(f) \mathrm{d} m$ $=\int f \mathrm{~d} m$, for all $t \in \mathbf{R}$. It follows from Example 2.3 that there is a unique topological index $\omega: \operatorname{Inv} C(G) \rightarrow \mathbf{R}$ such that $\omega(f)=\int f^{-1} D f \mathrm{~d} m$, for all $f \in$ $\operatorname{TP}(G) \cap \operatorname{Inv} C(G)$ (uniqueness uses the density of $\operatorname{TP}(G)$ in $C(G)$ ). Since $\omega\left(\varepsilon_{x}\right)=$ $\int \bar{\varepsilon}_{x} D\left(\varepsilon_{x}\right) \mathrm{d} m=\int \bar{\varepsilon}_{x} \varphi(x) \varepsilon_{x} \mathrm{~d} m=\varphi(x) \int 1 \mathrm{~d} m=\varphi(x)$, it follows that $\omega\left(\varepsilon_{x}\right) \geq 0$, for all $x \in \Gamma^{+}$.

Suppose now $E: \operatorname{TP}(G) \rightarrow C(G)$ is another skew-adjoint $G$-invariant derivation such that $\omega(f)=\int f^{-1} E f \mathrm{~d} m$, for all $f \in \operatorname{TP}(G) \cap \operatorname{Inv} C(G)$. Since $E \alpha_{g}\left(\varepsilon_{x}\right)=$ $\alpha_{g} E\left(\varepsilon_{x}\right)$, we have $\alpha_{g}(f)=\overline{g(x)} f$, for all $g \in G$, where $f=E\left(\varepsilon_{x}\right)$. Hence, $\alpha_{g}\left(f \bar{\varepsilon}_{x}\right)=$ $f \bar{\varepsilon}_{x}$, for all $g \in G$. This implies that $f \bar{\varepsilon}_{x}$ is a constant function and therefore $f=c(x) \varepsilon_{x}$, for some element $c(x) \in \mathbf{C}$. Thus, $E\left(\varepsilon_{x}\right)=c(x) \varepsilon_{x}$, for all $x \in \Gamma$. Since $\varphi(x)=\omega\left(\varepsilon_{x}\right)=\int \bar{\varepsilon}_{x} E\left(\varepsilon_{x}\right) \mathrm{d} m=\int \bar{\varepsilon}_{x} c(x) \varepsilon_{x} \mathrm{~d} m=c(x)$, we have $D\left(\varepsilon_{x}\right)=E\left(\varepsilon_{x}\right)$, for all $x \in \Gamma$, and therefore $D=E$.

Finally, suppose that $\omega^{\prime}: \operatorname{Inv} C(G) \rightarrow \mathbf{R}$ is another nonzero topological index for which $\omega^{\prime}\left(\varepsilon_{x}\right) \geq 0$, for all $x \in \Gamma^{+}$. Then the map $\varphi^{\prime}: \Gamma \rightarrow \mathbf{R}, x \mapsto \omega^{\prime}\left(\varepsilon_{x}\right)$, is a nonzero positive homomorphism. If $\varphi^{\prime}(z)=0$, then for any element $x \in \Gamma^{+}$, we would have $x \leq N z$, for some positive integer $N$, and therefore $0 \leq \varphi^{\prime}(x) \leq$ $N \varphi^{\prime}(z)=0$, giving $\varphi^{\prime}(x)=0$ and therefore implying the contradictory conclusion that $\varphi^{\prime}$ is identically zero. To avoid this we must conclude that $s=\varphi^{\prime}(z) \neq 0$. It follows that the map $\varphi^{\prime} / s$ is a positive homomorphism on $\Gamma$ mapping $z$ onto 1 , which implies $\varphi^{\prime} / s=\varphi$; that is, $\varphi^{\prime}=s \varphi$. Now let $f$ be an arbitrary element of $\operatorname{Inv} C(G)$. Then, by Theorem 2.5, there exists an element $x \in \Gamma$ and a function $h \in C(G)$ such that $f=\varepsilon_{x} e^{h}$. Hence, $\omega^{\prime}(f)=\omega^{\prime}\left(\varepsilon_{x}\right)+\omega^{\prime}\left(e^{h}\right)=\varphi^{\prime}(x)=s \varphi(x)=s \omega\left(\varepsilon_{x}\right)=s \omega(f)$. Thus, $\omega^{\prime}=s \omega$. 
Let us note explicitly for later use that if we choose a nonzero positive homomorphism $\varphi: \Gamma \rightarrow \mathbf{R}$, then we may choose $\omega$ in the theorem to satisfy the condition $\omega\left(\varepsilon_{x}\right)=\varphi(x)$, for all $x \in \Gamma$. Hence, $D\left(\varepsilon_{x}\right)=\varphi(x) \varepsilon_{x}$, for all $x \in \Gamma$.

We shall say in this case that $\omega$ is the topological index on $C(G)$ associated to $\varphi$ and $D$ is the derivation associated to $\varphi$.

Another item of terminology: Given an order relation $\leq$ on a discrete abelian group $\Gamma$ making it an ordered group, we shall say that a topological index $\omega$ : Inv $C(\hat{\Gamma}) \rightarrow \mathbf{R}$ is distinguished (relative to the ordered group $(\Gamma, \leq)$ ) provided $\omega\left(\varepsilon_{x}\right) \geq 0$, for all $x \in \Gamma^{+}$.

We illustrate these ideas in the following example.

Example 2.7. Let $\theta_{1}, \ldots, \theta_{N}$ be real numbers that are independent over $\mathbf{Z}$; that is, we suppose that if $\sum_{n=1}^{N} a_{n} \theta_{n}=0$, where $a_{1}, \ldots, a_{N}$ are integers, then we must have $a_{1}=\cdots=a_{N}=0$. Let $\Gamma$ be the ordered subgroup $\mathbf{Z} \theta_{1}+\cdots+\mathbf{Z} \theta_{N}$ of $\mathbf{R}$. Of course, in this case, $\hat{\Gamma}=\mathbf{T}^{N}$, where the pairing between $\Gamma$ and $\mathbf{T}^{N}$ is given by $\left(\left(a_{j}\right),\left(\lambda_{j}\right)\right) \mapsto \lambda_{1}^{a_{1}} \cdots \lambda_{N}^{a_{N}}$. Hence, if $x=a_{1} \theta_{1}+\cdots+a_{N} \theta_{N}$, then $\varepsilon_{x}=z_{1}^{a_{1}} \cdots z_{N}^{a_{N}}$, where $z_{1}, \ldots, z_{N}$ are the natural coordinate functions on $\mathbf{T}^{N}$.

In this case the unique (up-to-a-positive-scalar-factor) nonzero positive homomorphism $\varphi$ from $\Gamma$ to $\mathbf{R}$ is the inclusion function. Hence, the derivation $D$ : $\mathrm{TP}\left(\mathbf{T}^{N}\right) \rightarrow C\left(\mathbf{T}^{N}\right)$ associated to $\varphi$ is given by

$$
D\left(z_{1}^{a_{i}} \cdots z_{N}^{a_{N}}\right)=\left(\sum_{n=1}^{N} a_{n} \theta_{n}\right) z_{1}^{a_{i}} \cdots z_{N}^{a_{N}} .
$$

It follows that

$$
D(f)=\theta_{1} z_{1} \frac{\partial f}{\partial z_{1}}+\cdots+\theta_{N} z_{N} \frac{\partial f}{\partial z_{N}}
$$

for all $f \in \operatorname{TP}\left(\mathbf{T}^{N}\right)$. Therefore, if $f$ is a nonvanishing element of $\operatorname{TP}\left(\mathbf{T}^{N}\right)$, its distinguished topological index is given by

$$
\omega(f)=\int f^{-1} \sum_{j=1}^{N} \theta_{j} z_{j} \frac{\partial f}{\partial z_{j}} \mathrm{~d} m
$$

where $m$ is the normalized Haar measure of $\mathbf{T}^{N}$.

Now we turn to topological index theory for the Shilov boundary of a bounded symmetric domain. First we need to recall some facts about such domains. We refer to the book [26] of $\mathrm{H}$. Upmeier for details.

In fact, since every bounded symmetric domain is biholomorphically equivalent to a symmetric ball, we shall, without loss of generality, confine our discussion to symmetric balls.

Let $N$ be a positive integer. A subset $D$ of $\mathbf{C}^{N}$ is a symmetric ball if there is a norm $\|\cdot\|$ on $\mathbf{C}^{N}$ such that

$$
D=\left\{p \in \mathbf{C}^{N} \mid\|p\|<1\right\}
$$

and, for all $p, q \in D$, there exists a bijection $g: D \rightarrow D$ such that $g$ and $g^{-1}$ are holomorphic and $g(p)=q$.

Let $\bar{D}$ be the closure of $D$ in $\mathbf{C}^{N}$ and, for $j=1, \ldots, N$, let $z_{j}: \bar{D} \rightarrow \mathbf{C}$ be the $j$ th-coordinate function restricted to $\bar{D}$. The subalgebra $P_{0}(\bar{D})$ of $C(\bar{D})$ generated by $z_{1}, \ldots, z_{N}$ is the polynomial algebra on $\bar{D}$. We denote its closure in $C(\bar{D})$ by $P(\bar{D})$. 
There exists a smallest closed subset $S$ of $\bar{D}$ having the property that, for all $f \in P(\bar{D})$, we have $\|f\|=|f(p)|$, for some element $p \in S$. We call $S$ the Shilov boundary of $D$ (it is contained in the topological boundary of $D$ ).

Let $G=\left\{g \in \operatorname{Inv} M_{N}(\mathbf{C}) \mid g(D)=D\right\}$. Then $G$ is a compact subgroup of Inv $M_{N}(\mathbf{C})$ that acts transitively on $S$ and by a theorem of Bott and Korányi, there is a unique regular probability measure $m$ on $S$ that is $G$-invariant in the sense that $m(g(E))=m(E)$, for all Borel subsets $E$ of $S$ and all elements $g \in G$. Moreover, the support of $m$ is equal to $S$. We call $m$ the Bott-Korányi measure.

The action of $G$ on $S$ induces an action on $C(S)$; that is, a homomorphism $\alpha: G \rightarrow$ Aut $C(S)$, given by

$$
\alpha_{g}(f)(p)=f\left(g^{-1}(p)\right) \quad(g \in G, p \in S),
$$

for all $f \in C(S)$.

Now let $z_{1}, \ldots, z_{N}$ denote the coordinate functions on $S$. A polynomial on $S$ is an element of the subalgebra of $C(S)$ generated by $z_{1}, \ldots, z_{N}$ and a rational function on $S$ is a function of the form $f=f_{1} / f_{2}$, where $f_{1}$ and $f_{2}$ are polynomials on $S$ and $f_{2}$ is nonvanishing on $S$.

Suppose now that $D$ is a tube-type domain (the technical definition of tube type is that the $\mathrm{JB}^{*}$-triple system associated to $D$ admits an invertible element; there is a vast variety of examples of such domains). Then there exists a polynomial $\Delta$ on $S$ (a Koecher norm) of modulus one such that $\Delta \bar{z}_{j}$ is a polynomial on $S$, for $j=1, \ldots, N$. By the Stone-Weierstrass theorem, the $*$-subalgebra of $C(S)$ generated by the coordinate functions is dense in $C(S)$. It follows from this that the algebra of rational functions on $S$ is dense in $C(S)$, since this algebra contains all the $z_{j}$ and their conjugates $\bar{z}_{j}$, because each element $\bar{z}_{j}=\Delta^{-1} f_{j}$, where $f_{j}$ is some polynomial.

Note that if $r$ is the rank of $D$, the polynomial $\Delta$ is homogeneous of degree $r$, so that $\Delta(\lambda p)=\lambda^{r} \Delta(p)$, for all $\lambda \in \mathbf{T}$ and $p \in S$.

Theorem 2.8. Let $D$ be a tube-type symmetric ball in $\mathbf{C}^{N}$ and let $S$ be the Shilov boundary of $D$. Then there is a unique topological index $\omega: \operatorname{Inv} C(S) \rightarrow \mathbf{R}$ such that

$$
\omega(f)=\frac{1}{r} \int f^{-1} \sum_{j=1}^{N} z_{j} \frac{\partial f}{\partial z_{j}} \mathrm{~d} m,
$$

for all nonvanishing rational functions $f$ on $S$, where $m$ is the Bott-Korányi measure on $S$ and $r$ is the rank of $D$.

Proof. Uniqueness of $\omega$ is obvious, given that the rational functions are dense in $C(S)$.

If $t \in \mathbf{R}$, we get an element $g_{t}$ in $G$, the group of elements $g$ in $\operatorname{Inv} M_{N}(\mathbf{C})$ such that $g(D)=D$, by setting $g_{t}(p)=e^{-i t} p$, for all $p \in S$. This gives a strongly continuous homomorphism

$$
\beta: \mathbf{R} \rightarrow \operatorname{Aut} C(S), \quad t \mapsto \beta_{t}=\alpha_{g_{t}},
$$

and since

$$
\int \beta_{t}(f) \mathrm{d} m=\int f \mathrm{~d} m \quad(f \in C(S), t \in \mathbf{R}),
$$


by $G$-invariance of $m$, it follows from Theorem 2.2 and the observations made in Example 2.3 that there is a unique topological index $\omega: \operatorname{Inv} C(S) \rightarrow \mathbf{R}$ such that

$$
r \omega(f)=\int f^{-1} D_{\beta} f \mathrm{~d} m \quad\left(f \in \operatorname{Inv} \mathcal{A}_{\beta}\right),
$$

where $D_{\beta}: \mathcal{A}_{\beta} \rightarrow C(S)$ is the skew-adjoint derivation in $C(S)$ associated to the flow $\beta$.

Now $\lim _{t \rightarrow 0}\left(\alpha_{g_{t}}\left(z_{j}\right)-z_{j}\right) / t=\lim _{t \rightarrow 0} z_{j}\left(e^{i t}-1\right) / t=i z_{j}$, for $j=1, \ldots, N$. Hence, the coordinate functions $z_{j}$ belong to the inverse-closed subalgebra $\mathcal{A}_{\beta}$ of $C(S)$ and $D_{\beta}\left(z_{j}\right)=z_{j}$, for $j=1, \ldots, N$. Since the derivation $E=\sum_{j=1}^{N} z_{j} \frac{\partial}{\partial z_{j}}$ also satisfies the condition $E\left(z_{j}\right)=z_{j}$, for $j=1, \ldots, N$, we have $D_{\beta}=E$ on the algebra of rational functions on $S$.

We call the topological index constructed in the preceding theorem the distinguished topological index of $C(S)$.

Example 2.9. Consider the linear space $M_{N}(\mathbf{C})$ of square complex matrices of order $N$, endowed with the operator norm. The open unit ball $D$ of $M_{N}(\mathbf{C})$ is a symmetric ball of tube type and in this case the Shilov boundary is the set of unitary operators $U_{N}$ and the Bott-Korányi measure $m$ on $U_{N}$ is the normalized Haar measure of $U_{N}$ [26. The Koecher norm is the determinant function $\Delta: U_{N} \rightarrow \mathbf{C}$ and the rank of $D$ is $N$.

Now let $\omega: \operatorname{Inv} C\left(U_{N}\right) \rightarrow \mathbf{R}$ be the distinguished topological index. Then, since $\sum_{i, j=1}^{N} z_{i j} \frac{\partial \Delta}{\partial z_{i j}}=N \Delta$, we get

$$
\omega(\Delta)=\frac{1}{N} \int \bar{\Delta} N \Delta \mathrm{d} m=1 .
$$

Now suppose that $f: U_{N} \rightarrow \mathbf{C}$ is any nonvanishing continuous function. Then, since $U_{N}$ is a compact connected group, it follows from Theorem 2.5 that there is an integer $n$ and a function $h \in C\left(U_{N}\right)$ such that $f=\Delta^{n} e^{h}$ (this uses the fact that the only continuous characters $\chi: U_{N} \rightarrow \mathbf{T}$ are the functions $\left.\Delta^{n}\right)$. Hence, $\omega(f)=n$.

\section{Toeplitz operators on Hilbert modules}

To motivate what follows and to set up basic notation that we shall need repeatedly in the sequel, we begin this section by recalling some elementary facts about Toeplitz operators on the Hardy space of the circle.

Let $L^{2}=L^{2}(\mathbf{T})$ and let $\mathbf{z}: \mathbf{T} \rightarrow \mathbf{C}$ be the inclusion function. As is customary, we refer to the orthonormal basis $\left(e_{n}\right)_{n \in \mathbf{Z}}$, where $e_{n}=\mathbf{z}^{n}$, as the standard basis of $L^{2}$. The Hardy Hilbert space $H^{2}$ on $\mathbf{T}$ is defined to be the closed linear span in $L^{2}$ of the elements $e_{n}(n \geq 0)$.

If $W \in \mathcal{B}\left(H^{2}\right)$ has matrix $\left(b_{m n}\right)$ relative to the basis $\left(e_{n}\right)_{n=0}^{\infty}$, then $W=$ $\sum_{m, n=0}^{\infty} b_{m n} e_{m n}$, where convergence is relative to the strict topology in $\mathcal{B}\left(H^{2}\right)=$ $M\left(\mathcal{K}\left(H^{2}\right)\right)$ (the multiplier algebra). Recall that $W$ is a Toeplitz operator if its matrix $\left(b_{m n}\right)$ is constant on diagonals; that is, if $b_{m+1, n+1}=b_{m n}$, for all $m, n \geq 0$. Equivalently, $\mathbf{S}^{*} W \mathbf{S}=W$, where $\mathbf{S} \in \mathcal{B}\left(H^{2}\right)$ is the (forward) unilateral shift on the basis $\left(e_{n}\right)_{n=0}^{\infty}$. 
If $P$ is the orthogonal projection of $L^{2}$ onto $H^{2}$, and $f \in L^{\infty}(\mathbf{T})$, the operator

$$
T_{f}: H^{2} \rightarrow H^{2}, \quad h \mapsto P(f h),
$$

is a Toeplitz operator and all Toeplitz operators are obtained in this way.

As discussed in the Introduction, we shall be concerned with a wide variety of generalizations of Toeplitz operators. We shall be most concerned with generalizations of those Toeplitz operators $T_{f}$ that have continuous symbol $f \in C(\mathbf{T})$, since we shall be interested in the index theory and generalized Toeplitz operators with continuous symbol are most amenable to the $\mathrm{C}^{*}$-algebra techniques that we shall use here.

In this section we shall develop some aspects of a theory of Toeplitz operators on the Hilbert module $H^{2} \otimes \mathcal{B}$ over a unital $\mathrm{C}^{*}$-algebra $\mathcal{B}$ and of the associated Laurent operators on the Hilbert module $L^{2} \otimes \mathcal{B}$.

Let $\mathcal{B}$ be a unital $\mathrm{C}^{*}$-algebra, let $H$ be a Hilbert space and let $H_{\mathcal{B}}$ be the Hilbert module $H \otimes \mathcal{B}$ over $\mathcal{B}$. Recall that, as a Banach space, $H \otimes \mathcal{B}$ is obtained by completing the inner product module $H \odot \mathcal{B}$ over $\mathcal{B}$. As a linear space, $H \odot \mathcal{B}$ is the linear space tensor product of $H$ and $\mathcal{B}$. This space is endowed with the unique right $\mathcal{B}$-module structure for which $(f \otimes b) b^{\prime}=f \otimes b b^{\prime}$, for all $f \in H$ and $b, b^{\prime} \in \mathcal{B}$. The $\mathcal{B}$-valued inner product on $H \odot \mathcal{B}$ is the unique one for which

$$
\left\langle f_{1} \otimes b_{1} \mid f_{2} \otimes b_{2}\right\rangle=\left\langle f_{1} \mid f_{2}\right\rangle b_{1}^{*} b_{2},
$$

for all $f_{1}, f_{2} \in H$ and $b_{1}, b_{2} \in \mathcal{B}$.

If $W \in \mathcal{B}(H)$ and $b \in \mathcal{B}$, there is a unique operator $W \hat{\otimes} b$ in the $\mathrm{C}^{*}$-algebra $\mathcal{L}\left(H_{\mathcal{B}}\right)$ of adjointable operators on $H_{\mathcal{B}}$ for which $(W \hat{\otimes} b)\left(f \otimes b^{\prime}\right)=W f \otimes b b^{\prime}$, for all $f \in H$ and $b^{\prime} \in \mathcal{B}$. Also, it is well known that there is a unique $*$-isomorphism $\varphi$ from the multiplier algebra $M(K(H) \otimes \mathcal{B})$ to $\mathcal{L}\left(H_{\mathcal{B}}\right)$ for which $\varphi(W \otimes b)=W \hat{\otimes} b$, for all $W \in \mathcal{K}(H)$ and $b \in \mathcal{B}$ and $\varphi(K(H) \otimes \mathcal{B})=\mathcal{K}\left(H_{\mathcal{B}}\right)$, the closed ideal in $\mathcal{L}\left(H_{\mathcal{B}}\right)$ of all compact elements. Henceforth, we shall use $\varphi$ to identify $M(K(H) \otimes \mathcal{B})$ and $\mathcal{L}\left(H_{\mathcal{B}}\right)$, and we shall therefore simply write $W \otimes b$ for $W \hat{\otimes} b$. Thus, we shall use the multiplier algebra picture whenever it is convenient.

Let $\left(f_{i}\right)_{i \in I}$ be an orthonormal basis of $H$. If $F$ is a finite nonempty subset of $I$, denote by $P_{F}$ the finite-rank projection $P_{F} \in \mathcal{K}(H)$ given by $P_{F}=\sum_{i \in F} f_{i i}$. The net $\left(\hat{P}_{F}\right)_{F}=\left(P_{F} \otimes 1\right)_{F}$ converges strictly to 1 in $\mathcal{L}\left(H_{B}\right)=M(\mathcal{K}(H) \otimes B)$, and if $W \in \mathcal{L}\left(H_{B}\right)$ we have $W=\lim _{F} \hat{P}_{F} W \hat{P}_{F}$ in the strict topology. However, it is easily verified that

$$
\hat{P}_{F} W \hat{P}_{F}=\sum_{i, j \in F} f_{i j} \otimes b_{i j},
$$

where $b_{i j}=\left\langle f_{i} \otimes 1 \mid W\left(f_{j} \otimes 1\right)\right\rangle$. Thus, we can write the equation

$$
W=\lim _{F} \hat{P}_{F} W \hat{P}_{F}
$$

as

$$
W=\sum_{i, j \in I} f_{i j} \otimes b_{i j}
$$

with convergence in the strict topology. If $W \in \mathcal{K}\left(H_{B}\right)=\mathcal{K}(H) \otimes B$, then convergence in the norm topology also holds. Of course, all this is well known, but since it is important for the sequel, it seemed desirable to give details and establish notation.

We call $\left(b_{i j}\right)_{i j}$ the operator matrix of $W$ relative to the orthonormal basis $\left(f_{i}\right)_{i}$. 
Now we specialize to the case where $H=H^{2}$. An operator $W \in \mathcal{L}\left(H_{\mathcal{B}}^{2}\right)$ can then be written $W=\sum_{m, n=0}^{\infty} e_{m n} \otimes b_{m n}$. If the matrix $\left(b_{m n}\right)$ of $W$ is constant on diagonals; that is, if $b_{m+1, n+1}=b_{m n}$, for all $m, n \geq 0$, we say $W$ is a $\mathcal{B}$-Toeplitz operator.

Observe that $W$ is a $\mathcal{B}$-Toeplitz operator if, and only if, $V^{*} W V=W$, where $V$ is the isometry $\mathbf{S} \otimes 1$ in $\mathcal{L}\left(H_{\mathcal{B}}^{2}\right)$. For, if $W=\sum_{m, n=0}^{\infty} e_{m n} \otimes b_{m n}$, we have

$$
V^{*} W V=\sum_{m, n=0}^{\infty} \mathbf{S}^{*} e_{m n} \mathbf{S} \otimes b_{m n}=\sum_{m, n=0}^{\infty} e_{m n} \otimes b_{m+1, n+1} .
$$

Thus, $V^{*} W V$ has operator matrix $\left(b_{m+1, n+1}\right)_{m, n=0}^{\infty}$. Hence, $V^{*} W V=W$ if, and only if, $b_{m+1, n+1}=b_{m n}$, for all $m, n \geq 0$.

If $W \in \mathcal{L}\left(L_{\mathcal{B}}^{2}\right)$, then we may write

$$
W=\sum_{m, n \in \mathbf{Z}} e_{m n} \otimes b_{m n} .
$$

The compression of $W$ to $H_{\mathcal{B}}^{2}$ is the adjointable operator $T_{W}$ in $\mathcal{L}\left(H_{\mathcal{B}}^{2}\right)$ defined by setting $T_{W}(f)=P(W f)$, for all $f \in H_{\mathcal{B}}^{2}$, where $P \in \mathcal{L}\left(L_{\mathcal{B}}^{2}\right)$ is the projection of $L_{\mathcal{B}}^{2}$ onto $H_{\mathcal{B}}^{2}$. (It is immediate that $T_{W^{*}}$ provides an adjoint for $T_{W}$.) The operator matrix expression for $T_{W}$ is obviously

$$
T_{W}=\sum_{m, n=0}^{\infty} e_{m n} \otimes b_{m n} .
$$

We say $W$ is a $\mathcal{B}$-Laurent operator on $L_{\mathcal{B}}^{2}$ if its operator matrix $\left(b_{m n}\right)_{m, n \in \mathbf{Z}}$ has constant diagonals. In this case, $T_{W}$ is clearly a $\mathcal{B}$-Toeplitz operator. We call $W$ the symbol of $T_{W}$. It is obvious from the matrix operator expressions for $W$ and $T_{W}$ that $T_{W}$ uniquely determines $W$; that is, if $W_{1}, W_{2}$ are $\mathcal{B}$-Laurent operators and $T_{W_{1}}=T_{W_{2}}$, then $W_{1}=W_{2}$.

We denote by $\mathbf{U}$ the operator $M_{\mathbf{z}} \otimes 1$ in $\mathcal{L}\left(L_{\mathcal{B}}^{2}\right)$, where $M_{\mathbf{z}}$ is the multiplication operator $f \mapsto \mathbf{z} f$ in $\mathcal{B}\left(L^{2}\right)$. It is clear that an operator $W \in \mathcal{L}\left(L_{\mathcal{B}}^{2}\right)$ is a $\mathcal{B}$-Laurent operator if, and only if, $W$ commutes with $\mathbf{U}$. Hence, since $\mathbf{U}$ is a unitary operator, the set of $\mathcal{B}$-Laurent operators is a $\mathrm{C}^{*}$-subalgebra $\mathcal{L}_{\mathcal{B}}$ of $\mathcal{L}\left(L_{\mathcal{B}}^{2}\right)$.

Clearly, $T_{\mathbf{U}}=\mathbf{S} \otimes 1$.

We shall say that a $\mathcal{B}$-Laurent operator $W \in \mathcal{L}\left(L_{\mathcal{B}}^{2}\right)$ is of analytic type if $H_{\mathcal{B}}^{2}$ is invariant for $W$; that is, $W\left(H_{\mathcal{B}}^{2}\right) \subseteq H_{\mathcal{B}}^{2}$.

We observe that $T_{W}$ commutes with $\mathbf{S} \otimes 1$ if, and only if, $W$ is of analytic type. To see this, let $\left(b_{n}\right)_{n \in \mathbf{Z}}$ be the sequence in $\mathcal{B}$ for which $b_{m n}=b_{m-n}$, for all $m, n \in \mathbf{Z}$, where

$$
W=\sum_{m, n \in \mathbf{Z}} e_{m n} \otimes b_{m n}
$$

is the operator matrix expression for $W$. A simple matrix calculation shows that $T_{W}$ commutes with $\mathbf{S} \otimes 1$, if, and only if, $b_{n}=0$, for all $n<0$. Hence, if $T_{W}$ and $\mathbf{S} \otimes 1$ commute and $r \geq 0$, the element

$$
W\left(e_{r} \otimes 1\right)=\sum_{m, n \in \mathbf{Z}} e_{m n}\left(e_{r}\right) \otimes b_{m-n}=\sum_{m \in \mathbf{Z}} e_{m} \otimes b_{m-r}
$$

belongs to $H_{\mathcal{B}}^{2}$, since $b_{m-r}=0$ for $m-r<0$. It follows that $W\left(H_{\mathcal{B}}^{2}\right) \subseteq H_{\mathcal{B}}^{2}$. Conversely, if this inclusion holds, then equation (3) (with $r=0$ ) implies that $b_{n}=0$, for all $n<0$, and therefore $T_{W}$ commutes with $\mathbf{S} \otimes 1$. 
We denote by $\mathcal{T}_{\mathcal{B}}$ the selfadjoint closed linear subspace of $\mathcal{L}\left(H_{\mathcal{B}}^{2}\right)$ consisting of the $\mathcal{B}$-Toeplitz operators on $H_{\mathcal{B}}^{2}$.

Theorem 3.1. Let $\mathcal{B}$ be a unital $C^{*}$-algebra. The map

$$
T: \mathcal{L}_{\mathcal{B}} \rightarrow \mathcal{T}_{\mathcal{B}}, \quad W \mapsto T_{W},
$$

is an isometric *-linear map.

If $W_{1}, W_{2} \in \mathcal{L}_{\mathcal{B}}$ and $W_{2}$ is of analytic type, then $T_{W_{1}} T_{W_{2}}=T_{W_{1} W_{2}}$.

Proof. It is clear that $T$ is an injective, norm-decreasing *-linear map.

Let $W \in \mathcal{L}_{\mathcal{B}}$. For $n \geq 0$, set $W_{n}=\mathbf{U}^{* n} T_{W} P \mathbf{U}^{n} \in \mathcal{L}\left(L_{B}^{2}\right)$ and note that $\left\|W_{n}\right\| \leq\left\|T_{W}\right\|$. If $f$ and $h$ are in the linear span of the elements $e_{m} \otimes b(m \in \mathbf{Z}$, $b \in \mathcal{B}$ ), then for all sufficiently large positive $n, \mathbf{U}^{* n} f$ and $\mathbf{U}^{* n} h$ belong to $H_{\mathcal{B}}^{2}$. Hence,

$$
\left\langle f \mid W_{n} h\right\rangle=\left\langle\mathbf{U}^{n} f \mid W \mathbf{U}^{n} h\right\rangle=\langle f \mid W h\rangle .
$$

It follows easily from this that $\langle f \mid W h\rangle=\lim _{n}\left\langle f \mid W_{n} h\right\rangle$, for all $f, h \in L_{B}^{2}$. In particular, $\|W h\|^{2}=\lim _{n}\left\langle W h \mid W_{n} h\right\rangle$ and therefore

$$
\|W h\|^{2} \leq\|W h\|\left\|T_{W}\right\|\|h\| \text {. }
$$

Hence, $\|W h\| \leq\left\|T_{W}\right\|\|h\|$. Thus, $\|W\| \leq\left\|T_{W}\right\|$ so that $\|W\|=\left\|T_{W}\right\|$.

The second statement in the theorem, although very useful, is trivially verified.

An obvious question is whether the map $T$ in the preceding theorem is surjective; that is, whether every $\mathcal{B}$-Toeplitz operator is the compression of a $\mathcal{B}$-Laurent operator. This is, of course, true in the case of Toeplitz operators on $H^{2}$, but we are unable to determine if it remains true in the general situation. The usual proof [9. pp. 338-339] for the classical situation does not extend (at least in any straightforward way), principally because of problems with whether relevant operators are adjointable.

In this connection one should note that Theorem 4.11 gives a partial answer to the preceding question.

We want to consider now the index theory of our generalized Toeplitz operators. First we need to recall some definitions and results on the Fredholm index associated to a trace.

Let $\mathcal{A}$ be a unital $\mathrm{C}^{*}$-algebra and let tr be a nonzero densely-defined trace on a proper closed ideal $\mathcal{K}$ of $\mathcal{A}$. We shall always write $L^{1}(\operatorname{tr})$ for the domain of definition of tr. An element $a \in \mathcal{A}$ is tr-Fredholm if there is an element $b \in \mathcal{A}$ such that $1-a b$ and $1-b a$ belong to $L^{1}(\operatorname{tr})$. In this case, we set

$$
\text { index }_{\operatorname{tr}}(a)=\operatorname{tr}(a b-b a) \text {. }
$$

It is shown in 17 that the following basic properties hold:

(1) If $a \in \mathcal{A}$, then $a$ is tr-Fredholm if, and only if, $a+\mathcal{K}$ is invertible in the quotient $\mathrm{C}^{*}$-algebra $\mathcal{A} / \mathcal{K}$.

(2) The set of tr-Fredholm elements of $\mathcal{A}$ is closed under multiplication. Moreover, if $a$ and $b$ are tr-Fredholm elements of $\mathcal{A}$, then

$$
\text { index }_{\mathrm{tr}}(a b)=\operatorname{index}_{\mathrm{tr}}(a)+\operatorname{index}_{\mathrm{tr}}(b) \text {. }
$$

(3) The map $a \mapsto \operatorname{index}_{\operatorname{tr}}(a)$ is locally constant.

(4) If $a$ is a tr-Fredholm element of $\mathcal{A}$ and $c \in \mathcal{K}$, then

$$
\text { index }_{\operatorname{tr}}(a+c)=\text { index }_{\operatorname{tr}}(a) \text {. }
$$


Note that index $\operatorname{tr}(a)$ is always a real number but that, unlike the classical Fredholm index, it may not be integer-valued.

Now let $H$ be a Hilbert space and $\mathcal{B}$ a unital $\mathrm{C}^{*}$-algebra and set $H_{\mathcal{B}}=H \otimes \mathcal{B}$. If $\tau$ is a tracial state of $\mathcal{B}$, there is a unique norm-bounded positive linear map $\iota \otimes \tau: \mathcal{K}(H) \otimes \mathcal{B} \rightarrow \mathcal{K}(H)$ such that $(\iota \otimes \tau)(W \otimes b)=\tau(b) W$, for all $W \in \mathcal{K}(H)$ and $b \in \mathcal{B}$. This enables us to define a lower semicontinuous function

$$
\operatorname{tr}_{\tau}: \mathcal{K}\left(H_{\mathcal{B}}\right)^{+} \rightarrow[0,+\infty], \quad W \mapsto \operatorname{Tr}((\iota \otimes \tau)(W)),
$$

on the closed ideal $\mathcal{K}\left(H_{\mathcal{B}}\right)$ of compact elements of $\mathcal{L}\left(H_{\mathcal{B}}\right)$. Here, and in the sequel, $\operatorname{Tr}$ denotes the standard trace on the ideal of compact operators of a Hilbert space.

If $\left(f_{i}\right)_{i \in I}$ is an orthonormal basis of $H$, and $W \in \mathcal{K}\left(H_{B}\right)^{+}$has operator matrix representation

$$
W=\sum_{i, j \in I} f_{i j} \otimes b_{i j}
$$

then

$$
(\iota \otimes \tau) W=\sum_{i, j \in I} f_{i j} \tau\left(b_{i j}\right),
$$

and therefore

$$
\operatorname{tr}_{\tau}(W)=\sum_{i \in I} \tau\left(b_{i i}\right) .
$$

It follows easily from this, using the fact that $\tau$ is a trace, that $\operatorname{tr}_{\tau}$ is also a trace (a well-known result).

Note that if $W$ belongs to the ideal $L^{1}(\operatorname{Tr})$ of trace-class operators on $H$ and if $b$ is an element of $\mathcal{B}$, then $W \otimes b$ belongs to the definition domain $L^{1}\left(\operatorname{tr}_{\tau}\right) \operatorname{of} \operatorname{tr}_{\tau}$ and $\operatorname{tr}_{\tau}(W \otimes b)=\operatorname{Tr}(W) \tau(b)$.

We call $\operatorname{tr}_{\tau}$ the trace on $\mathcal{K}\left(H_{\mathcal{B}}\right)$ induced by the tracial state $\tau$ on $\mathcal{B}$. Note that $\operatorname{tr}_{\tau}$ is densely defined on $\mathcal{K}\left(H_{\mathcal{B}}\right)$, since $L^{1}(\operatorname{Tr}) \odot \mathcal{B}$ is dense in $\mathcal{K}(H) \otimes \mathcal{B}$. To avoid clumsy notation, we denote by index ${ }_{\tau}$ the index associated to $\operatorname{tr}_{\tau}$ on the $\operatorname{tr}_{\tau}$-Fredholm operators in $\mathcal{L}\left(H_{\mathcal{B}}\right)$.

Since $C(\mathbf{T}, \mathcal{B})=C(\mathbf{T}) \otimes \mathcal{B}$, there is a unique $*$-isomorphism $\rho$ from $C(\mathbf{T}, \mathcal{B})$ onto the $\mathrm{C}^{*}$-subalgebra $C^{*}\left(M_{\mathbf{z}}\right) \otimes \mathcal{B}$ of $\mathcal{L}_{\mathcal{B}}$ sending $\mathbf{z} \otimes b$ onto $\mathbf{U}(1 \otimes b)$, for all $b \in \mathcal{B}$, where $\mathbf{z} \otimes b$ is the map $\mathbf{T} \rightarrow \mathcal{B}, \lambda \mapsto \lambda b$. Henceforth, if $f \in C(\mathbf{T}, \mathcal{B})$, we shall write $T_{f}$ for $T_{\rho(f)}$. This gives us an isometric $*$-linear map $T: f \mapsto T_{f}$ from $C(\mathbf{T}, \mathcal{B})$ to $\mathcal{T}_{\mathcal{B}}$. We call the elements in $\mathcal{T}_{\mathcal{B}}$ of the form $T_{f}$, where $f \in C(\mathbf{T}, \mathcal{B}), \mathcal{B}$ Toeplitz operators with continuous symbols. We denote by $\mathcal{A}(\mathcal{B})$ the $\mathrm{C}^{*}$-subalgebra of $\mathcal{L}\left(H_{\mathcal{B}}^{2}\right)$ generated by all $T_{f}(f \in C(\mathbf{T}, \mathcal{B}))$.

If $\mathcal{B}=\mathbf{C}$, then $H_{\mathcal{B}}^{2}=H^{2}$ and the $\mathcal{B}$-Toeplitz operators are just the classical Toeplitz operators. In this case $\mathcal{A}(\mathbf{C})$ is generated as a $\mathrm{C}^{*}$-algebra by $\mathbf{S}$.

Theorem 3.2. Let $\mathcal{B}$ be a unital $C^{*}$-algebra. Then $\mathcal{A}(\mathcal{B})=\mathcal{A}(\mathbf{C}) \otimes \mathcal{B}$. There is a unique $*$-homomorphism $\pi: \mathcal{A}(\mathcal{B}) \rightarrow C(\mathbf{T}, \mathcal{B})$ such that $\pi\left(T_{f}\right)=f$, for all $f \in C(\mathbf{T}, \mathcal{B})$. The kernel of $\pi$ is equal to $\mathcal{K}\left(H_{\mathcal{B}}^{2}\right)$.

Let $\tau$ be a tracial state on $\mathcal{B}$ and let $\operatorname{tr}_{\tau}$ be the induced trace on $\mathcal{K}\left(H_{\mathcal{B}}^{2}\right)$. Then if $f \in C(\mathbf{T}, \mathcal{B}), T_{f}$ is a $\operatorname{tr}_{\tau}-$ Fredholm operator if, and only if, $f$ is invertible.

Proof. Since the elements $\mathbf{z}^{n} \otimes b$, where $n \in \mathbf{Z}$ and $b \in \mathcal{B}$, have closed linear span $\mathcal{C}=C(\mathbf{T}, \mathcal{B})$, the elements $T_{\mathbf{z} \otimes b}=\mathbf{S} \otimes b$ generate $\mathcal{A}(\mathcal{B})$. Hence, $\mathcal{A}(\mathcal{B})=\mathcal{A}(\mathbf{C}) \otimes \mathcal{B}$. It follows that $\mathcal{K}\left(H_{\mathcal{B}}^{2}\right)=\mathcal{K}\left(H^{2}\right) \otimes \mathcal{B}$ is a closed ideal in $\mathcal{A}(\mathcal{B})$. 
The map $\rho: \mathcal{C} \rightarrow \mathcal{A}(\mathcal{B}) / \mathcal{K}\left(H_{\mathcal{B}}\right), f \mapsto T_{f}+\mathcal{K}\left(H_{\mathcal{B}}^{2}\right)$, is clearly a norm-decreasing *-linear map. The set $\mathcal{C}_{0}$ of all $f \in \mathcal{C}$ such that

$$
\rho(h f)=\rho(h) \rho(f) \quad \text { and } \quad \rho(f h)=\rho(f) \rho(h),
$$

for all $h \in \mathcal{C}$, is a $C^{*}$-subalgebra of $\mathcal{C}$. Since $T_{\mathbf{U}(1 \otimes b)}=T_{\mathbf{z} \otimes b}=\mathbf{S} \otimes b$ commutes with $\mathbf{S} \otimes 1, \mathbf{U}(1 \otimes b)$ is an analytic-type $\mathcal{B}$-Laurent operator, from which it follows immediately that the first equality in equation (4) holds for $f=\mathbf{z} \otimes b$ and for all $h \in \mathcal{C}$.

If $b \in \mathcal{B}$, we claim that $f=\mathbf{z} \otimes b$ belongs to $\mathcal{C}_{0}$. Again suppose $h \in \mathcal{C}$ and write $T_{h}=\sum_{m, n=0}^{\infty} e_{m n} \otimes b_{m n}$. Then $T_{f} T_{h}-T_{f h}=-\sum_{n=0}^{\infty} e_{0 n} \otimes b b_{-1, n}$. Hence, $T_{f} T_{h}-T_{f h}=\left(e_{00} \otimes 1\right)\left(T_{f} T_{h}-T_{f h}\right)$, so $T_{f} T_{h}-T_{f h}$ belongs to $\mathcal{K}\left(H_{\mathcal{B}}^{2}\right)$, since $e_{00} \otimes 1 \in \mathcal{K}\left(H_{\mathcal{B}}^{2}\right)$. Hence, for all $b \in \mathcal{B}, \mathbf{z} \otimes b \in \mathcal{C}_{0}$, and since these elements generate $\mathcal{C}$, we have $\mathcal{C}_{0}=\mathcal{C}$. Therefore, $\rho$ is a $*$-homomorphism. Since the elements $T_{f}$ $(f \in \mathcal{C})$ generate $\mathcal{A}(\mathcal{B})$, the map $\rho$ is surjective.

To see that $\rho$ is injective, suppose $T_{f} \in \mathcal{K}\left(H_{\mathcal{B}}^{2}\right)$ and let $T_{f}=\sum_{m, n=0}^{\infty} e_{m n} \otimes b_{m n}$. Then, for all $\tau \in \mathcal{B}^{*},(\iota \otimes \tau)\left(T_{f}\right)=\sum_{m, n=0}^{\infty} e_{m n} \tau\left(b_{m n}\right)$ is a compact classical Toeplitz operator on $H^{2}$ and therefore is equal to zero. Hence, $\tau\left(b_{m n}\right)=0$, for all $m, n \geq 0$. Since $\tau$ was an arbitrary element of $\mathcal{B}^{*}$, this shows that all $b_{m n}=0$ and therefore $T_{f}=0$. Hence, $f=0$. Thus, $\rho$ is a $*$-isomorphism.

Therefore, composing the quotient map $\mathcal{A}(\mathcal{B}) \rightarrow \mathcal{A}(\mathcal{B}) / \mathcal{K}\left(H_{\mathcal{B}}^{2}\right)$ with $\rho^{-1}$ gives a (unique) $*$-homomorphism $\pi: \mathcal{A}(\mathcal{B}) \rightarrow C(\mathbf{T}, \mathcal{B})$ such that $\pi\left(T_{f}\right)=f$, for all $f \in \mathcal{C}$. Clearly, $\operatorname{ker}(\pi)=\mathcal{K}\left(H_{\mathcal{B}}^{2}\right)$.

Now let $\tau$ be a tracial state on $\mathcal{B}$. If $f \in \mathcal{C}$, then $T_{f}$ is a $\operatorname{tr}_{\tau}$-Fredholm operator if, and only if, $T_{f}$ is invertible modulo $\mathcal{K}\left(H_{\mathcal{B}}^{2}\right)$ (since $\mathcal{K}\left(H_{B}^{2}\right)$ is equal to the norm closure of $\left.L^{1}\left(\operatorname{tr}_{\tau}\right)\right)$; using $\pi$ and its properties, it's clear that this is equivalent to invertibility of $f$ in $\mathcal{C}$.

An abstract index theorem was proved in 20] that we shall need to use now. We therefore recall some details from [20].

If $\mathcal{C}$ is a unital $\mathrm{C}^{*}$-algebra, an indicial triple for $\mathcal{C}$ is a triple $\Omega=(\mathcal{L}, F, \operatorname{tr})$, where

(1) $\mathcal{L}$ is a unital $\mathrm{C}^{*}$-algebra containing $\mathcal{C}$ as a unital $\mathrm{C}^{*}$-subalgebra;

(2) $F$ is a selfadjoint unitary in $\mathcal{L}$;

(3) $\operatorname{tr}: \mathcal{L}^{+} \rightarrow[0,+\infty]$ is a lower semicontinuous trace on $\mathcal{L}$;

(4) the $*$-subalgebra $\mathcal{C}_{0}$ of all elements $f$ in $\mathcal{C}$ such that $[F, f]$ belongs to $L^{1}(\operatorname{tr})$ is dense in $\mathcal{C}$.

Given such an indicial triple, there is a unique locally constant function

$$
\omega_{\Omega}: \operatorname{Inv} \mathcal{C} \rightarrow \mathbf{R}, \quad f \mapsto \omega_{\Omega}(f)
$$

such that

$$
\omega_{\Omega}(f)=\operatorname{tr}\left(f^{-1}[F, f]\right) / 2,
$$

for all elements $f$ in $\mathcal{C}_{0}$ that are invertible in $\mathcal{C}$. Moreover, $\omega_{\tau}$ is a topological index on $\mathcal{C}$. (The factor $1 / 2$ is a normalization constant inserted to give the right formula in index theorems.)

Set $P=(F+1) / 2$, so $P$ is a projection. Let $\mathcal{A}$ be the $\mathrm{C}^{*}$-algebra generated by all $\operatorname{PfP}(f \in \mathcal{C})$ and denote by $\operatorname{tr}_{\Omega}$ the restriction to $\mathcal{A}^{+}$of the trace tr. It is shown in 20] that $P f P$ is a $\operatorname{tr}_{\Omega}$-Fredholm element in $\mathcal{A}$ provided $f$ is invertible in $\mathcal{C}$ and in this case

$$
\text { index }_{\operatorname{tr}_{\Omega}}(P f P)=-\omega_{\Omega}(f) .
$$


Now suppose that $\mathcal{B}$ is a unital $\mathrm{C}^{*}$-algebra admitting a tracial state $\tau$ and set $\mathcal{C}=C(\mathbf{T}, \mathcal{B})$, identified with the $\mathrm{C}^{*}$-subalgebra $C^{*}\left(M_{\mathbf{z}}\right) \otimes \mathcal{B}$ of $\mathcal{L}\left(L_{\mathcal{B}}^{2}\right)$. Let $P$ be the projection of $L_{\mathcal{B}}^{2}$ onto $H_{\mathcal{B}}^{2}$ and let $F$ be the selfadjoint unitary $2 P-1$. Let $\operatorname{tr}_{\tau}$ be the trace on $\mathcal{K}\left(L_{\mathcal{B}}^{2}\right)$ induced by $\tau$ and extend it trivially to a trace on $\mathcal{L}\left(L_{\mathcal{B}}^{2}\right)$ by setting $\operatorname{tr}_{\tau}(W)=+\infty$ for all positive operators $W \in \mathcal{L}\left(H_{\mathcal{B}}^{2}\right) \backslash \mathcal{K}\left(L_{\mathcal{B}}^{2}\right)$. Then the extended trace, still denoted $\operatorname{tr}_{\tau}$, remains lower semicontinuous and its definition ideal remains unchanged. The triple $\Omega_{\tau}=\left(\mathcal{L}\left(L_{\mathcal{B}}^{2}\right), F, \operatorname{tr}_{\tau}\right)$ is an indicial triple for $\mathcal{C}$. To see this we need only show the $*$-subalgebra $\mathcal{C}_{0}$ of all elements $f$ in $\mathcal{C}$ such that $[F, f]$ belongs to $L^{1}\left(\operatorname{tr}_{\tau}\right)$ is dense in $\mathcal{C}$. Therefore, since the elements $\mathbf{z} \otimes b$ generate $\mathcal{C}$, we need only show $[F, \mathbf{U}(1 \otimes b)]=2[P, \mathbf{U}(1 \otimes b)]$ belongs to $L^{1}\left(\operatorname{tr}_{\tau}\right)$. However, since $P=P_{0} \otimes 1$, where $P_{0}$ is the projection of $L^{2}$ onto $H^{2}$, we have $[P, \mathbf{U}(1 \otimes b)]=\left[P_{0}, M_{\mathbf{z}}\right] \otimes b$, so $[P, \mathbf{U}(1 \otimes b)] \in L^{1}\left(\operatorname{tr}_{\tau}\right)$, since $\left[P_{0}, M_{\mathbf{z}}\right]$ is a finite-rank operator.

We call $\Omega_{\tau}$ the indicial triple on $C(\mathbf{T}, \mathcal{B})$ associated to the tracial state $\tau$ on $\mathcal{B}$ and we call the function $\omega_{\tau}=\omega_{\Omega_{\tau}}$ on $\operatorname{Inv} C(\mathbf{T}, \mathcal{B})$ the topological index function associated to $\tau$. Note $\omega_{\tau}$ is nontrivial, since $\omega_{\tau}(\mathbf{z} \otimes 1)=1$. This follows from the calculation $\omega_{\tau}(\mathbf{z} \otimes 1)=\operatorname{tr}_{\tau}\left(\left(M_{\overline{\mathbf{z}}} \otimes 1\right)\left[P, M_{\mathbf{z}} \otimes 1\right]\right)=\operatorname{tr}_{\tau}\left(M_{\overline{\mathbf{z}}}\left[P_{0}, M_{\mathbf{z}}\right] \otimes 1\right)=$ $\operatorname{Tr}\left(M_{\overline{\mathbf{z}}}\left[P_{0}, M_{\mathbf{z}}\right]\right) \tau(1)=1$.

We now prove a general index theorem for $\mathcal{B}$-Toeplitz operators with continuous symbols. We shall apply this theorem to other classes of Toeplitz operators on Hardy spaces of ordered groups and of bounded symmetric domains in later sections.

We need some notation for this theorem. We write $\operatorname{TP}(\mathbf{T}, \mathcal{B})$ for the dense *subalgebra $\operatorname{TP}(\mathbf{T}) \odot \mathcal{B}$ of $C(\mathbf{T}, \mathcal{B})=C(\mathbf{T}) \otimes \mathcal{B}$. Every element $f \in \operatorname{TP}(\mathbf{T}, \mathcal{B})$ can be written in the form

$$
f=\sum_{n \in \mathbf{Z}} \mathbf{z}^{n} \otimes b_{n}
$$

where the $b_{n}$ are uniquely determined elements of $\mathcal{B}$, all but a finite number of which are equal to zero. (Thus, the elements of $\mathrm{TP}(\mathbf{T}, \mathcal{B})$ are "trigonometric polynomials" with coefficients in $\mathcal{B}$.)

Theorem 3.3. Let $\mathcal{B}$ be a unital $C^{*}$-algebra and let $\tau$ be a tracial state on $\mathcal{B}$. If $f \in C(\mathbf{T}, \mathcal{B})$, then the $\mathcal{B}$-Toeplitz operator $T_{f}$ is $\operatorname{tr}_{\tau}$-Fredholm if, and only if, $f$ is invertible in $C(\mathbf{T}, \mathcal{B})$ and in this case,

$$
\operatorname{index}_{\tau}\left(T_{f}\right)=-\omega_{\tau}(f) .
$$

Moreover, $\omega_{\tau}: \operatorname{Inv} C(\mathbf{T}, \mathcal{B}) \rightarrow \mathbf{R}$ is the unique topological index such that

$$
\omega_{\tau}(f)=\int(\iota \otimes \tau)\left(f^{-1} D f\right) \mathrm{d} m,
$$

for all $f \in \operatorname{TP}(\mathbf{T}, \mathcal{B}) \cap \operatorname{Inv} C(\mathbf{T}, \mathcal{B})$, where $m$ is the normalised Haar measure on $\mathbf{T}$ and $D: \operatorname{TP}(\mathbf{T}, \mathcal{B}) \rightarrow C(\mathbf{T}, \mathcal{B})$ is the unique skew-adjoint derivation such that

$$
D\left(\mathbf{z}^{n} \otimes b\right)=n \mathbf{z}^{n} \otimes b \quad(n \in \mathbf{Z}, b \in \mathcal{B}) .
$$

Proof. First we remark that we can identify $\mathcal{L}\left(H_{\mathcal{B}}^{2}\right)$ with the $\mathrm{C}^{*}$-subalgebra $P \mathcal{L}\left(L_{\mathcal{B}}^{2}\right) P$ of $\mathcal{L}\left(L_{\mathcal{B}}^{2}\right)$, where $P$ is the projection of $L_{\mathcal{B}}^{2}$ onto $H_{\mathcal{B}}^{2}$, via the obvious *-isomorphism. Under this identification, $T_{f}$ is identified with $P f P$. Hence, by the index theorem for the indicial triple $\Omega_{\tau}=\left(\mathcal{L}\left(L_{\mathcal{B}}^{2}\right), 2 P-1, \operatorname{tr}_{\tau}\right)$ stated in the discussion preceding this theorem, if $f \in \operatorname{Inv} C(\mathbf{T}, \mathcal{B}), T_{f}$ is a $\operatorname{tr}_{\tau}$-Fredholm operator and $\operatorname{index}_{\tau}\left(T_{f}\right)=\operatorname{index}_{\operatorname{tr}_{\Omega_{\tau}}}(P f P)=-\omega_{\tau}(f)$. 
Now let $D: \operatorname{TP}(\mathbf{T}, \mathcal{B}) \rightarrow C(\mathbf{T}, \mathcal{B})$ be the unique linear map such that $D\left(\mathbf{z}^{n} \otimes b\right)$ $=n \mathbf{z}^{n} \otimes b$, for all $n \in \mathbf{Z}$ and $b \in \mathcal{B}$. It is immediate that $D$ is a skew-adjoint derivation. To prove equation (5) holds, it suffices to show

$$
\operatorname{tr}_{\tau}\left(f_{1}\left[F, f_{2}\right]\right)=2 \int(\iota \otimes \tau)\left(f_{1} D f_{2}\right) \mathrm{d} m
$$

for all $f_{1}$ and $f_{2}$ in $\operatorname{TP}(\mathbf{T}, \mathcal{B})$. Here, in the left hand side of this equation, we are identifying $f_{1}$ and $f_{2}$ with their canonical images in $\mathcal{L}\left(L_{\mathcal{B}}^{2}\right)$. One should explicitly note too that we are using the fact that $\operatorname{tr}_{\tau}\left(\cdot\left[F, f_{2}\right]\right)$ is a continuous linear functional on $C(\mathbf{T}, \mathcal{B})$, since $\left[F, f_{2}\right]$ belongs to $L^{1}\left(\operatorname{tr}_{\tau}\right)$. Clearly, we may make a further reduction and suppose that, for $j=1,2$, we have $f_{j}=\mathbf{z}^{n_{j}} \otimes b_{j}$, for some integers $n_{1}, n_{2}$ and some elements $b_{1}, b_{2}$ in $\mathcal{B}$. Then, if $F_{0}=2 P_{0}-1$, where $P_{0}$ is the projection of $L^{2}$ onto $H^{2}$, we have

$$
\begin{gathered}
\operatorname{tr}_{\tau}\left(f_{1}\left[F, f_{2}\right]\right)=\operatorname{Tr}\left((\iota \otimes \tau)\left(\mathbf{z}^{n_{1}}\left[F_{0}, \mathbf{z}^{n_{2}}\right] \otimes b_{1} b_{2}\right)\right) \\
=\operatorname{Tr}\left(\mathbf{z}^{n_{1}}\left[F_{0}, \mathbf{z}^{n_{2}}\right]\right) \tau\left(b_{1} b_{2}\right)=\operatorname{Tr}\left(\mathbf{z}^{n_{1}} n_{2} \mathbf{z}^{n_{2}-1}\left[F_{0}, \mathbf{z}\right]\right) \tau\left(b_{1} b_{2}\right) .
\end{gathered}
$$

Now an easy calculation shows that $\left[F_{0}, \mathbf{z}\right]=2 e_{0,-1}$ and therefore

Hence,

$$
\begin{aligned}
& \operatorname{Tr}\left(\mathbf{z}^{n_{1}} n_{2} \mathbf{z}^{n_{2}-1}\left[F_{0}, \mathbf{z}\right]\right)=2 n_{2} \operatorname{Tr}\left(e_{n_{1}+n_{2}-1,-1}\right) \\
& =2 n_{2}\left\langle e_{n_{1}+n_{2}-1} \mid e_{-1}\right\rangle=2 n_{2} \int \mathbf{z}^{n_{1}+n_{2}} \mathrm{~d} m .
\end{aligned}
$$

$$
\begin{gathered}
\operatorname{tr}_{\tau}\left(f_{1}\left[F, f_{2}\right]\right)=2 n_{2} \int \mathbf{z}^{n_{1}+n_{2}} \mathrm{~d} m \tau\left(b_{1} b_{2}\right) \\
=2 \int(\iota \otimes \tau)\left(\left(\mathbf{z}^{n_{1}} \otimes b_{1}\right) D\left(\mathbf{z}^{n_{2}} \otimes b_{2}\right)\right) \mathrm{d} m=2 \int(\iota \otimes \tau)\left(f_{1} D f_{2}\right) \mathrm{d} m,
\end{gathered}
$$

as required.

We shall illustrate this theory with many examples in the sequel. For now, we remark only that in the case $\mathcal{B}=\mathbf{C}$, the result reduces to the Gohberg-Krein theorem. In this case, the only possibility for $\tau$ is the identity map on C. Since the corresponding topological index function $\omega_{\tau}$ is equal to 1 on $\mathbf{z}$ and is a locally constant homomorphism, it is clear that, for all $f \in \operatorname{Inv} C(\mathbf{T}), \omega_{\tau}(f)$ is equal to the winding number of $f$ with respect to the origin. Of course, in this case the $\operatorname{tr}_{\tau}$-index is just the usual Fredholm index on $\mathcal{B}\left(H^{2}\right)$. Hence, our result says here that if $f \in C(\mathbf{T})$, the Toeplitz operator $T_{f}$ is a Fredholm operator if, and only if, $f$ never vanishes and in this case, the Fredholm index of $T_{f}$ is equal to minus the winding number of $f$ around the origin.

\section{Toeplitz elements in $\mathrm{C}^{*}$-Algebras and Representation theory}

Our next theorem (Theorem 4.1) is fundamental for the results of this paper. We shall use it to show that Toeplitz operators on Hardy spaces of ordered groups and of tube-type bounded symmetric domains can be represented as $\mathcal{B}$-Toeplitz operators for a suitable $\mathrm{C}^{*}$-algebra $\mathcal{B}$.

First we need some preliminaries on terminology and notation.

Let $\mathcal{A}$ be a unital $\mathrm{C}^{*}$-algebra and $V$ a proper isometry in $\mathcal{A}$ (so $V^{*} V=1$ and $V V^{*} \neq 1$ ). In analogy with $\mathcal{B}$-Toeplitz operators, we can define the elements of the closed, selfadjoint linear space

$$
\mathcal{T}_{V}=\left\{W \in \mathcal{A} \mid V^{*} W V=W\right\}
$$


as $V$-Toeplitz elements of $\mathcal{A}$ and the elements of the closed algebra

$$
\mathcal{T}_{V}^{a}=\{W \in \mathcal{A} \mid V W=W V\}
$$

as the analytic-type $V$-Toeplitz elements.

We denote by $\mathcal{K}_{V}$ the closed ideal in $\mathcal{A}$ generated by the projection $Q_{V}=1-V V^{*}$ and by $\mathcal{B}_{V}$ the $\mathrm{C}^{*}$-algebra $Q_{V} \mathcal{A} Q_{V}$, with unit $Q_{V}$.

Now set $Q_{n}=1-V^{n} V^{* n}$, for all $n \geq 0$. Then $\left(Q_{n}\right)_{n}$ forms a sequence of projections in $\mathcal{K}_{V}$ that is increasing, since $Q_{n+1}=Q_{n}+V^{n} Q_{V} V^{* n}$.

At the present level of generality, the definition of $V$-Toeplitz elements is a fairly vacuous one, but we shall see in the next theorem that it has real content when suitable extra conditions are imposed on $V$. Moreover, these extra conditions are not very restrictive and we shall exhibit in the sequel vast classes of algebras and isometries that satisfy them.

A natural first condition to impose on $V$ is that the set $\mathcal{T}_{V}$ of $V$-Toeplitz elements generate $\mathcal{A}$ as a $\mathrm{C}^{*}$-algebra. If this is not the case, we can simply restrict to the $\mathrm{C}^{*}$-algebra generated by $\mathcal{T}_{V}$. If $W \in \mathcal{T}_{V}$, then $V^{*} W V=W$ and therefore $W+\mathcal{K}_{V}$ commutes with $V+\mathcal{K}_{V}$, since $V+\mathcal{K}_{V}$ is a unitary in $\mathcal{A} / \mathcal{K}_{V}$. It follows that if $\mathcal{T}_{V}$ generates $\mathcal{A}$, then $V+\mathcal{K}_{V}$ belongs to the centre of $\mathcal{A} / \mathcal{K}_{V}$, a result we shall use often.

Theorem 4.1. Let $V$ be a proper isometry in a unital $C^{*}$-algebra $\mathcal{A}$ and suppose that $\mathcal{T}_{V}$ generates $\mathcal{A}$. The following are equivalent conditions:

(1)

$$
\lim _{n \rightarrow \infty}\left[W V^{n}, V\right]=0 \quad(W \in \mathcal{A})
$$

$$
\lim _{n \rightarrow \infty} Q_{V} W V^{n}=0 \quad(W \in \mathcal{A}) .
$$

(3) The sequence of projections $\left(Q_{n}\right)_{n=0}^{\infty}$ forms an approximate unit for $\mathcal{K}_{V}$, where $Q_{n}=1-V^{n} V^{* n}$, for all $n \geq 0$.

(4) There is a $*$-homomorphism $\psi: \mathcal{A} \rightarrow \mathcal{L}\left(H_{\mathcal{B}_{V}}^{2}\right)$ that maps $\mathcal{K}_{V}$ isomorphically onto the ideal $\mathcal{K}\left(H_{\mathcal{B}_{V}}^{2}\right)$ of compact elements of $\mathcal{L}\left(H_{\mathcal{B}_{V}}^{2}\right)$ and is such that $\psi(V)=\mathbf{S} \otimes 1$ and $\psi(b)=e_{00} \otimes b$, for all $b \in \mathcal{B}_{V}$.

Moreover, if Condition (4) holds, then $\psi$ is unique and if $W \in \mathcal{T}_{V}$, then $\psi(W)$ is a $\mathcal{B}_{V}$-Toeplitz operator in $\mathcal{L}\left(H_{\mathcal{B}_{V}}^{2}\right)$.

The map $\psi$ is injective if, and only if, $\mathcal{K}_{V}$ is essential as a closed ideal in $\mathcal{A}$.

Proof. If Condition (2) holds, then $\lim _{n} W V^{n}=0$ for all elements $W$ of the ideal generated algebraically by $Q_{V}$ and therefore for all elements $W$ of its closure $\mathcal{K}_{V}$. Hence, $\lim _{n} W V^{n} V^{* n}=0$ and therefore $W=\lim _{n} W Q_{n}$. Therefore, the sequence $\left(Q_{n}\right)_{n}$ forms an approximate unit for $\mathcal{K}_{V}$. Thus, Condition (2) implies (3) and the reverse implication is evident, so that these conditions are equivalent.

Now suppose that Condition (3) holds; we shall show this implies that (4) holds. If $N \geq 1$, let $M_{N}$ denote the linear space of all elements of the form $W=\sum_{m, n=0}^{N} e_{m n} \otimes b_{m n}$, where $b_{m n} \in \mathcal{B}_{V}$. We define a map $\varphi_{N}: M_{N} \rightarrow \mathcal{A}$ by setting $\varphi_{N}(W)=\sum_{m, n=0}^{N} V^{m} b_{m n} V^{* n}$. An elementary calculation, using the easily-checked formula $Q_{V} V^{* n} V^{m} Q_{V}=\delta_{m n} Q_{V}$, and the fact that $M_{N}$ is a $\mathrm{C}^{*}$ subalgebra of $\mathcal{K}\left(H^{2}\right) \otimes \mathcal{B}_{V}$, shows that $\varphi_{N}$ is a $*$-homomorphism. Moreover, $\varphi_{N}$ is 
injective: for if $\sum_{m, n=0}^{N} V^{m} b_{m n} V^{* n}=0$, then for all $0 \leq k, l \leq N$,

$$
\sum_{m, n=0}^{N} Q_{V} V^{* k} V^{m} Q_{V} b_{m n} Q_{V} V^{* n} V^{l} Q_{V}=0 ;
$$

that is, $b_{k l}=Q_{V} b_{k l} Q_{V}=0$. It follows that $\varphi_{N}$ is isometric. Now the sequence $\left(M_{N}\right)_{N}$ is increasing and its union $\bigcup_{N} M_{N}$ is a dense $*$-subalgebra of $\mathcal{K}\left(H^{2}\right) \otimes \mathcal{B}_{V}$; moreover, $\varphi_{N+1}$ is equal to $\varphi_{N}$ when restricted to $M_{N}$. Hence, we may extend the $\varphi_{N}$ s to get an isometric $*$-homomorphism $\varphi: \mathcal{K}\left(H^{2}\right) \otimes \mathcal{B}_{V} \rightarrow \mathcal{A}$.

Since $\varphi\left(\mathcal{K}\left(H^{2}\right) \otimes \mathcal{B}_{V}\right)$ contains $Q_{V}$, because $\varphi\left(e_{00} \otimes 1\right)=Q_{V}$, and is contained in $\mathcal{K}_{V}$, to show it is equal to $\mathcal{K}_{V}$ it suffices now to show it is an ideal in $\mathcal{K}_{V}$. Selfadjointness of $\varphi\left(\mathcal{K}\left(H^{2}\right) \otimes \mathcal{B}_{V}\right)$ implies it suffices to show $\varphi\left(\mathcal{K}\left(H^{2}\right) \otimes \mathcal{B}_{V}\right)$ is a left ideal. To see this we need only show that if $W \in \varphi\left(\mathcal{K}\left(H^{2}\right) \otimes \mathcal{B}_{V}\right)$ and $T \in \mathcal{K}_{V}$, then $Q_{N} T W \in \varphi\left(\mathcal{K}\left(H^{2}\right) \otimes \mathcal{B}_{V}\right)$. Without loss of generality, we may suppose $W=V^{m} b V^{* n}$, for some $m, n \geq 0$ and some element $b \in \mathcal{B}_{V}$. We complete this part of the proof by induction on $N$. Clearly, if $N=0$, then, since $Q_{0}=0$, we have $Q_{0} T W \in \varphi\left(\mathcal{K}\left(H^{2}\right) \otimes \mathcal{B}_{V}\right)$ trivially. Suppose now we know $Q_{N} T W \in$ $\varphi\left(\mathcal{K}\left(H^{2}\right) \otimes \mathcal{B}_{V}\right)$, for some value $N$. Since $Q_{N+1}=Q_{N}+V^{N} Q_{V} V^{* N}$, we have $Q_{N+1} T W=Q_{N} T W+V^{N} Q_{V} V^{* N} T V^{m} b Q_{V} V^{* n}$. Therefore, since $Q_{N} T W$ and $V^{N} Q_{V} V^{* N} T V^{m} b Q_{V} V^{* n}$ belong to $\varphi\left(\mathcal{K}\left(H^{2}\right) \otimes \mathcal{B}_{V}\right)$, because $Q_{V} V^{* N} T V^{m} b Q_{V} \in$ $\mathcal{B}_{V}$, we have $Q_{N+1} T W \in \varphi\left(\mathcal{K}\left(H^{2}\right) \otimes \mathcal{B}_{V}\right)$, completing the induction.

The $*$-isomorphism $\varphi^{-1}: \mathcal{K}_{V} \rightarrow \mathcal{K}\left(H^{2}\right) \otimes \mathcal{B}_{V}$ induces a $*$-homomorphism $\psi$ : $\mathcal{A} \rightarrow M\left(\mathcal{K}\left(H^{2}\right) \otimes \mathcal{B}_{V}\right)$. Clearly, $\psi(V)=\mathbf{S} \otimes 1$, since

$$
\begin{gathered}
\psi(V)\left(e_{m n} \otimes b\right)=\psi(V) \psi\left(V^{m} b V^{* n}\right)=\psi\left(V^{m+1} b V^{* n}\right) \\
=e_{m+1, n} \otimes b=(\mathbf{S} \otimes 1)\left(e_{m n} \otimes b\right),
\end{gathered}
$$

for all $m, n \geq 0$ and all $b \in \mathcal{B}_{V}$. Thus, Condition (4) holds.

Now suppose that Condition (4) holds and let $\psi_{1}, \psi_{2}: \mathcal{A} \rightarrow \mathcal{L}\left(H_{\mathcal{B}_{V}}^{2}\right)$ be *homomorphisms mapping $\mathcal{K}_{V}$ isomorphically onto $\mathcal{K}\left(H_{\mathcal{B}_{V}}^{2}\right)$ and mapping $V$ onto $\mathbf{S} \otimes 1$ and each element $b$ of $\mathcal{B}_{V}$ onto $e_{00} \otimes b$; we shall show $\psi_{1}=\psi_{2}$. First observe that, for $j=1,2, \psi_{j}\left(V^{m} b V^{* n}\right)=\left(\mathbf{S}^{m} \otimes 1\right)\left(e_{00} \otimes b\right)\left(\mathbf{S}^{* n} \otimes 1\right)=e_{m n} \otimes b$, for all $m, n \geq$ 0 and all $b \in \mathcal{B}_{V}$. Hence, $\psi_{1}=\psi_{2}$ on $\mathcal{K}_{V}$. Therefore, if $W \in \mathcal{A}$ and $W_{0} \in \mathcal{K}_{V}$, then $\psi_{1}\left(W W_{0}\right)=\psi_{2}\left(W W_{0}\right)$ and therefore $\left(\psi_{1}(W)-\psi_{2}(W)\right) \psi_{1}\left(W_{0}\right)=0$. Thus, $\left(\psi_{1}(W)-\psi_{2}(W)\right) \mathcal{K}\left(H_{\mathcal{B}_{V}}^{2}\right)=0$. Since the ideal $\mathcal{K}\left(H_{\mathcal{B}_{V}}^{2}\right)$ is essential in $\mathcal{L}\left(H_{\mathcal{B}_{V}}^{2}\right)$, this implies that $\psi_{1}(W)=\psi_{2}(W)$. Therefore $\psi_{1}=\psi_{2}$.

The sequence $\left(\left(1-\mathbf{S}^{n} \mathbf{S}^{* n}\right) \otimes 1\right)_{n=0}^{\infty}$ clearly forms an approximate unit for $\mathcal{K}\left(H^{2}\right)$ $\otimes \mathcal{B}_{V}$, from which it follows that the sequence of projections $Q_{n}=$ $\psi_{j}\left(\left(1-\mathbf{S}^{n} \mathbf{S}^{* n}\right) \otimes 1\right)$ forms an approximate unit for $\mathcal{K}_{V}$. Hence, Condition (3) holds, if Condition (4) holds. Therefore, Conditions (3) and (4) are equivalent.

It is obvious that the map $\psi$ is injective if, and only if, $\mathcal{K}_{V}$ is essential as a closed ideal in $\mathcal{A}$.

We finish the proof by showing the equivalence of Condition (1) with Conditions (2)-(4). If (3) holds, then $\lim _{n} W V^{n}=0$, for all $W \in \mathcal{K}_{V}$. Now if $W \in \mathcal{A}$, then $\left[W+\mathcal{K}_{V}, V+\mathcal{K}_{V}\right]=0$, since $V+\mathcal{K}_{V}$ is in the centre of $\mathcal{A} / \mathcal{K}_{V}$ because $\mathcal{T}_{V}$ generates $\mathcal{A}$, as we observed in the remarks preceding this theorem. Hence, $\lim _{n}\left[W V^{n}, V\right]=\lim _{n}[W, V] V^{n}=0$. Thus, Condition (3) implies (1).

Now suppose that Condition (1) holds; we shall show that this implies (2). If $W \in \mathcal{A}$, then $\lim _{n} Q_{V} W V^{n+1}=\lim _{n}\left(Q_{V}\left[W V^{n}, V\right]+Q_{V} V W V^{n}\right)=0$, since $\lim _{n}\left[W V^{n}, V\right]=0$ and $Q_{V} V=0$. Hence, $\lim _{n} Q_{V} W V^{n}=0$. 
Corollary 4.2. If the equivalent conditions of the theorem hold, then

$$
\left\|W+\mathcal{K}_{V}\right\|=\lim _{n \rightarrow \infty}\left\|V^{* n} W V^{n}\right\| \quad(W \in \mathcal{A}) .
$$

Proof. Since $\left(Q_{n}\right)$ is an approximate unit for $\mathcal{K}_{V}$, if $W \in \mathcal{A}$, then

$$
\left\|W+\mathcal{K}_{V}\right\|=\lim _{n}\left\|W\left(1-Q_{n}\right)\right\|=\lim _{n}\left\|W V^{n} V^{* n}\right\| .
$$

However, $\left\|W V^{n} V^{* n}\right\| \geq\left\|V^{* n} W V^{n}\right\| \geq\left\|V^{* n} W V^{n}+\mathcal{K}_{V}\right\|=\left\|W+\mathcal{K}_{V}\right\|$ (the equality is a consequence of the fact that $V+\mathcal{K}_{V}$ is a unitary of $\mathcal{A} / \mathcal{K}_{V}$ ). Equation (6) follows immediately.

Corollary 4.3. If the equivalent conditions of the theorem hold, $\mathcal{T}_{V} \cap \mathcal{K}_{V}=0$. Moreover, if in addition $\mathcal{A}$ acts irreducibly on a Hilbert space $H, \mathcal{T}_{V} \cap \mathcal{K}(H)=0$.

Proof. If $W \in \mathcal{T}_{V} \cap \mathcal{K}_{V}$, then, since $W=V^{* n} W V^{n}$, for all $n \geq 0$, we have

$$
\|W\|=\lim _{n \rightarrow \infty}\left\|V^{* n} W V^{n}\right\|=\left\|W+\mathcal{K}_{V}\right\|=0
$$

and therefore, $W=0$.

Now suppose $\mathcal{A}$ acts irreducibly on a Hilbert space $H$. If $\mathcal{T}_{V} \cap \mathcal{K}(H) \neq 0$, then irreducibility implies that $\mathcal{K}(H) \subseteq \mathcal{A}$ and therefore $\mathcal{K}(H) \subseteq \mathcal{K}_{V}$. Since $\mathcal{T}_{V} \cap \mathcal{K}_{V}=0$, therefore $\mathcal{T}_{V} \cap \mathcal{K}(H)=0$, a contradiction.

We call the $*$-homomorphism $\psi: \mathcal{A} \rightarrow \mathcal{L}\left(H_{\mathcal{B}_{V}}^{2}\right)$ of Theorem 4.1 the representation of $\mathcal{A}$ associated to the isometry $V$.

The "asymptotic-commuting" condition $\lim _{n}\left[W V^{n}, V\right]=0$ in the theorem is a natural one in the context of Toeplitz theory and is satisfied in a vast variety of examples. One might initially suppose that a simpler, more natural, "asymptoticcommuting" condition would be that $\lim _{n}\left[W, V^{n}\right]=0$. However, if $W$ satisfies this condition, we have $\lim _{n} V^{* n}\left[W, V^{n}\right]=0$ and therefore $W=\lim _{n} V^{* n} W V^{n}$. Hence, $V^{*} W V=W$. Since $W=V V^{*}$ does not satisfy the Toeplitz condition $V^{*} W V=W$ if $V V^{*} \neq 1$, it is clear the condition $\lim _{n}\left[W, V^{n}\right]=0$ does not hold in general.

Results related to the representation in Theorem 4.1 were proved in 2 by C.A. Berger, L.A. Coburn and A. Lebow and in [18 by the author. Although both earlier results are more specialized in their scope, do not involve Hilbert modules and do not represent abstract Toeplitz elements as Toeplitz operators, nevertheless there are points of contact, and these earlier results provided the inspiration for formulating and proving Theorem 4.1.

Corollary 4.3 rules out fitting the class of Bergman-Toeplitz operators on the open unit disc $D$ in $\mathbf{C}$ within our framework, at least not in any straightforward manner: Let $m$ be normalized planar Lebesgue measure on $D$ and recall that the Bergman space $H$ of $D$ is the set of all analytic functions on $D$ that are squareintegrable relative to $m$. If $f \in L^{\infty}(D, m)$, then the Bergman-Toeplitz operator $T_{f}$ is the compression to $H$ of the multiplication operator $M_{f}: h \mapsto f h$ on $L^{2}(D, m)$. Let $\mathcal{A}$ be the (unital) $\mathrm{C}^{*}$-algebra generated by all Bergman-Toeplitz operators $T_{f}$ on $H$ with $f \in C(\bar{D})$. This algebra contains $\mathcal{K}(H)[22$ and therefore acts irreducibly on $H$. Moreover, if $f$ is a function in $C(\bar{D})$ that vanishes on the boundary, then $T_{f}$ is a compact operator 22. It follows that there are nonzero compact BergmanToeplitz operators in $\mathcal{A}$. Hence, by the preceding theorem, there is no proper isometry $V$ in $\mathcal{A}$ such that $\mathcal{T}_{V}$ is equal to the class of all Bergman-Toeplitz operators $T_{f}$ with $f \in C(\bar{D})$ and for which the equivalent conditions of Theorem 4.1 hold. 
Of course, as the preceding observations illustrate, Toeplitz operators on the Bergman space of the disc behave very differently from Toeplitz operators on the Hardy space of its boundary. The intuition behind the theory developed here is that we are looking for classes of operators that behave like the Toeplitz operators on the boundary; therefore, it is quite natural that Bergman-Toeplitz operators would not appear to fall within the ambit of this theory.

Theorem 4.4. Let $V$ be a proper isometry in a unital $C^{*}$-algebra $\mathcal{A}$ such that $\mathcal{T}_{V}$ generates $\mathcal{A}$ and $\left(1-V^{n} V^{* n}\right)_{n}$ is an approximate unit for $\mathcal{K}_{V}$. Let $\pi: \mathcal{A} \rightarrow \mathcal{C}$ be a surjective $*$-homomorphism from $\mathcal{A}$ onto a unital $C^{*}$-algebra $\mathcal{C}$ such that $\operatorname{ker}(\pi)=$ $\mathcal{K}_{V}$. Then

$$
\|\pi(W)\|=\|W\| \quad\left(W \in \mathcal{T}_{V}\right) .
$$

Also, $\mathcal{T}_{V}+\mathcal{K}_{V}$ is closed in $\mathcal{A}$.

The following are equivalent statements:

(1) For all elements $W \in \mathcal{A}$, the sequence $\left(V^{* n} W V^{n}\right)_{n}$ is convergent.

(2) $\mathcal{A}=\mathcal{T}_{V}+\mathcal{K}_{V}$.

(3) There exists a $*$-linear map $T: \mathcal{C} \rightarrow \mathcal{A}, f \mapsto T_{f}$, such that $T(\mathcal{C})=\mathcal{T}_{V}$ and $\pi\left(T_{f}\right)=f$, for all $f \in \mathcal{C}$.

If Condition (3) holds, then $T$ is unique, positive and isometric. Also,

$$
T_{f \pi(W)}=T_{f} W \quad\left(f \in \mathcal{C}, W \in \mathcal{T}_{V}^{a}\right) .
$$

Proof. If $W \in \mathcal{T}_{V}$, then $\|\pi(W)\|=\left\|W+\mathcal{K}_{V}\right\|=\lim _{n \rightarrow \infty}\left\|V^{* n} W V^{n}\right\|=\|W\|$, by Equation (6). Since $\mathcal{T}_{V}$ is closed in $\mathcal{A}$ and therefore complete, it follows that $\pi\left(\mathcal{T}_{V}\right)$ is complete and therefore closed in $\mathcal{C}$. Hence, $\mathcal{T}_{V}+\mathcal{K}_{V}=\pi^{-1}\left(\pi\left(\mathcal{T}_{V}\right)\right)$ is closed in $\mathcal{A}$.

If $W \in \mathcal{T}_{V}$ and $K \in \mathcal{K}_{V}$, then $V^{* n}(W+K) V^{n}=W+V^{* n} K V^{n}$; hence, by Equation (6), $\lim _{n} V^{* n}(W+K) V^{n}=W$. This shows that Condition (2) implies (1).

Suppose conversely that Condition (1) holds; we shall show that (2) holds. In this case, if $W \in \mathcal{A}$, set $\tilde{W}=\lim _{n} V^{* n} W V^{n}$. Then $\tilde{W} \in \mathcal{T}_{V}$ and $\pi(\tilde{W})=$ $\lim _{n} \pi(V)^{* n} \pi(W) \pi(V)^{n}=\pi(W)$, so $W-\tilde{W} \in \mathcal{K}_{V}$. Hence, $W \in \mathcal{T}_{V}+\mathcal{K}_{V}$.

If Condition (3) holds and $W \in \mathcal{A}$, then $K=W-T_{\pi(W)} \in \mathcal{K}_{V}$ and therefore $W \in \mathcal{T}_{V}+\mathcal{K}_{V}$; hence, Condition (2) holds.

Assume now that (2) holds; we shall show that (3) holds. In this case the restriction $\pi: \mathcal{T}_{V} \rightarrow \mathcal{C}$ is a surjective isometric $*$-linear map and therefore admits an inverse $T: \mathcal{C} \rightarrow \mathcal{A}$ that is also $*$-linear and isometric and for which we have $T(\mathcal{C})=\mathcal{T}_{V}$.

It is well known (and easy to see) that a unital norm-decreasing $*$-linear map between unital $\mathrm{C}^{*}$-algebras is automatically positive. Hence, in particular, the map $T: \mathcal{C} \rightarrow \mathcal{A}$ is positive.

Now suppose $T: \mathcal{C} \rightarrow \mathcal{A}$ is any linear right inverse map for $\pi$ such that $T(\mathcal{C})=$ $\mathcal{T}_{V}$. If $W \in \mathcal{A}$ and $W$ commutes with $V$, then $T_{f \pi(W)}=T_{f} W$, for all $f \in \mathcal{C}$. For, $\pi\left(T_{f \pi(W)}-T_{f} W\right)=f \pi(W)-f \pi(W)=0$, so $T_{f \pi(W)}-T_{f} W \in \mathcal{K}_{V}$. But it is clear that $\mathcal{T}_{V} W \subseteq \mathcal{T}_{V}$, so that $T_{f \pi(W)}-T_{f} W \in \mathcal{T}_{V}$. Hence, since $\mathcal{T}_{V} \cap \mathcal{K}_{V}=0$, $T_{f \pi(W)}=T_{f} W$

Now we show uniqueness of $T$. More precisely, let $T^{1}$ and $T^{2}$ be two $*$-linear maps from $\mathcal{C}$ to $\mathcal{A}$ that are right inverses of $\pi$ with range equal to $\mathcal{T}_{V}$; we shall show that $T^{1}=T^{2}$. Set $K_{f}=T_{f}^{1}-T_{f}^{2}$, for $f \in \mathcal{C}$. Then $K_{f \pi\left(V^{n}\right)}=K_{f} V^{n}$, for all $n \geq 0$, and, by taking adjoints, we see also that $K_{\pi\left(V^{* n}\right) f}=V^{* n} K_{f}$. Since $\pi\left(K_{f}\right)=$ 
$\pi\left(T_{f}^{1}\right)-\pi\left(T_{f}^{2}\right)=f-f=0$, we have $K_{f} \in \mathcal{K}_{V}$. Hence, $0=\lim _{n \rightarrow \infty} V^{n *} K_{f} V^{n}=$ $\lim _{n \rightarrow \infty} K_{\pi\left(V^{n *}\right) f \pi\left(V^{n}\right)}=K_{f}$. Therefore, $K_{f}=0$ and so $T_{f}^{1}=T_{f}^{2}$.

The conditions in the preceding theorem are not always easy to check. However, in the case that the algebra of "symbols" $\mathcal{C}$ is commutative - an important case for our theory - we have the following result:

Corollary 4.5. If $\mathcal{T}_{V}^{a}$ generates $\mathcal{A}$ and $\mathcal{C}$ is commutative, then the three equivalent conditions of the theorem hold.

Proof. Let $\mathcal{A}_{0}$ denote the linear span of elements of the form $W_{1} W_{2}^{*}$, where $W_{1}, W_{2}$ $\in \mathcal{T}_{V}^{a}$. Since $\mathcal{A}_{0}$ is selfadjoint, so is $\mathcal{A}_{0}+\mathcal{K}_{V}$. If $W_{j} \in \mathcal{T}_{V}^{a}$, for $j=1,2,3,4$, then $W_{1} W_{2}^{*} W_{3} W_{4}^{*}=W_{1} W_{3}\left(W_{4} W_{2}\right)^{*}+W_{1}\left[W_{2}^{*}, W_{3}\right] W_{4}^{*}$. Since $\left[W_{2}^{*}, W_{3}\right] \in \mathcal{K}_{V}$, this shows $W_{1} W_{2}^{*} W_{3} W_{4}^{*} \in \mathcal{A}_{0}+\mathcal{K}_{V}$. It follows that $\mathcal{A}_{0}+\mathcal{K}_{V}$ is a subalgebra of $\mathcal{A}$. Hence, the closure of $\mathcal{A}_{0}+\mathcal{K}_{V}$ is a $\mathrm{C}^{*}$-algebra containing $\mathcal{T}_{V}^{a}$ and therefore it is equal to $\mathcal{A}$, since $\mathcal{T}_{V}^{a}$ generates $\mathcal{A}$. Let $W_{1}, W_{2} \in \mathcal{T}_{V}^{a}$. Since $W_{1} W_{2}^{*}=\left[W_{1}, W_{2}^{*}\right]+W_{2}^{*} W_{1}$ and $W_{2}^{*} W_{1} \in \mathcal{T}_{V}$, therefore $\mathcal{A}_{0} \subseteq \mathcal{T}_{V}+\mathcal{K}_{V}$. This, together with the fact that $\mathcal{T}_{V}+\mathcal{K}_{V}$ is closed in $\mathcal{A}$, implies that $\mathcal{A}=\mathcal{T}_{V}+\mathcal{K}_{V}$.

Note that the hypothesis of the preceding corollary implies the algebra $\mathcal{T}_{V}^{a}$ is commutative. For, if $W_{1}, W_{2} \in \mathcal{T}_{V}^{a}$, then, by Equation (6),

$$
\begin{aligned}
0= & \left\|W_{1} W_{2}-W_{2} W_{1}+\mathcal{K}_{V}\right\|=\lim _{n}\left\|V^{* n}\left(W_{1} W_{2}-W_{2} W_{1}\right) V^{n}\right\| \\
& =\lim _{n}\left\|V^{* n} V^{n}\left(W_{1} W_{2}-W_{2} W_{1}\right)\right\|=\left\|W_{1} W_{2}-W_{2} W_{1}\right\| .
\end{aligned}
$$

Hence, $W_{1} W_{2}-W_{2} W_{1}=0$.

We call the map $T: \mathcal{C} \rightarrow \mathcal{A}$ in Theorem 4.4 the $V$-related right inverse of $\pi$ or, less precisely, the Toeplitz map related to $V$.

The properties enjoyed by $T$ are, of course, very similar to those enjoyed by the usual Toeplitz map $C(\mathbf{T}) \rightarrow \mathcal{B}\left(H^{2}\right), f \mapsto T_{f}$, and this is the reason for our terminology.

It is natural to ask the question whether, if $V$ is a proper isometry in a unital $\mathrm{C}^{*}$-algebra $\mathcal{A}$ such that $\mathcal{T}_{V}$ generates $\mathcal{A}$ and $\left(1-V^{n} V^{* n}\right)_{n}$ is an approximate unit for $\mathcal{K}_{V}$, it is automatic that $\mathcal{A}=\mathcal{T}_{V}+\mathcal{K}_{V}$. The answer is no. To see this, let $\mathcal{A}$ be the $\mathrm{C}^{*}$-algebra generated by all Toeplitz operators $T_{f}$ on $H^{2}$, for arbitrary $f \in L^{\infty}$. Let $V$ be the proper isometry $\mathbf{S}$. The closed ideal in $\mathcal{A}$ generated by $Q=1-V V^{*}$ is clearly the ideal $\mathcal{K}\left(H^{2}\right)$ of compact operators, and therefore the sequence $\left(1-V^{n} V^{* n}\right)_{n}$ is an approximate unit for $\mathcal{K}_{V}$. There exists a $*$ homomorphism $\pi: \mathcal{A} \rightarrow L^{\infty}$ such that $\pi\left(T_{f}\right)=f$, for all $f \in L^{\infty}$, 8 , Theorem 7.11]. Since $\mathcal{A}$ is primitive (it acts irreducibly on $H^{2}$ ), so $\operatorname{ker}(\pi) \cap \mathcal{K}\left(H^{2}\right) \neq 0$ and therefore, since $\mathcal{K}\left(H^{2}\right)$ is a simple $\mathrm{C}^{*}$-algebra, $\mathcal{K}\left(H^{2}\right) \subseteq \operatorname{ker}(\pi)$. If now $\mathcal{A}=$ $\mathcal{T}_{V}+\mathcal{K}_{V}$, then $W \in \operatorname{ker}(\pi)$ implies $W=T_{f}+K$, for some element $f \in L^{\infty}$ and some operator $K \in \mathcal{K}\left(H^{2}\right)$. Hence, $T_{f}=W-K \in \operatorname{ker}(\pi)$ and therefore $f=\pi\left(T_{f}\right)=0$. This implies $W=K \in \mathcal{K}\left(H^{2}\right)$. Hence, $\operatorname{ker}(\pi)=\mathcal{K}\left(H^{2}\right)$. It follows, since $\pi\left(T_{f} T_{g}-T_{f g}\right)=f g-f g=0$, that $T_{f} T_{g}-T_{f g} \in \mathcal{K}\left(H^{2}\right)$, for all $f, g \in L^{\infty}$. But this is false [8, p. 204]. Hence, we cannot have $\mathcal{A}=\mathcal{T}_{V}+\mathcal{K}_{V}$.

We shall say that a proper isometry $V$ in a unital $\mathrm{C}^{*}$-algebra $\mathcal{A}$ is pivotal if $\mathcal{T}_{V}$ generates $\mathcal{A}$ and, for each element $W \in \mathcal{T}_{V}$, there exists a sequence of elements $\left(W_{n}\right)_{n}$ in $\mathcal{T}_{V}^{a}$ and positive integers $m_{n}$ such that $W=\lim _{n \rightarrow \infty} V^{* m_{n}} W_{n}$.

Theorem 4.6. Let $V$ be a pivotal isometry in a unital $C^{*}$-algebra $\mathcal{A}$. Then the sequence $\left(1-V^{n} V^{* n}\right)_{n}$ is an approximate unit for $\mathcal{K}_{V}$ and $\mathcal{A}=\mathcal{T}_{V}+\mathcal{K}_{V}$. 
Proof. The hypothesis clearly implies that $\mathcal{T}_{V}^{a}$ generates $\mathcal{A}$. To see that the sequence $\left(1-V^{n} V^{* n}\right)_{n}$ is an approximate unit for $\mathcal{K}_{V}$, it suffices, by Theorem 4.1, to show that $\lim _{n \rightarrow \infty} Q_{V} W V^{n}=0$, for all elements $W \in \mathcal{A}$. Clearly, to show this we may suppose that $W=V^{* m_{N}} W_{N} \cdots V^{* m_{1}} W_{1}$, for some elements $W_{1}, \ldots, W_{N} \in \mathcal{T}_{V}^{a}$ and positive integers $m_{1}, \ldots, m_{N}$ (the reason for writing the subscripts in this order will be apparent in a moment). Choose $n>m_{1}+\cdots+m_{N}$. Then

$$
\begin{aligned}
& Q_{V} W V^{n}=Q_{V} V^{* m_{N}} W_{N} \cdots V^{* m_{1}} V^{n} W_{1} \\
& =Q_{V} V^{* m_{N}} W_{N} \cdots V^{* m_{2}} W_{2} V^{n-m_{1}} W_{1} \\
& =Q_{V} V^{* m_{N}} W_{N} \cdots V^{n-m_{1}-m_{2}} W_{2} W_{1} \\
& =\cdots=Q_{V} V^{n-m_{1}-\cdots-m_{n}} W_{N} \cdots W_{1}=0,
\end{aligned}
$$

since $Q_{V} V=0$. Hence, $\lim _{n \rightarrow \infty} Q_{V} W V^{n}=0$, as required.

To show $\mathcal{T}_{V}+\mathcal{K}_{V}$ is equal to $\mathcal{A}$, it suffices to show it is a $\mathrm{C}^{*}$-subalgebra of $\mathcal{A}$, since $\mathcal{T}_{V}$ generates $\mathcal{A}$. Hence, since $\mathcal{T}_{V}+\mathcal{K}_{V}$ is a selfadjoint closed linear subspace of $\mathcal{A}$, by Theorem 4.4 it suffices to show that $\mathcal{T}_{V}+\mathcal{K}_{V}$ is multiplicatively closed. Let $W$ and $U$ be elements of $\mathcal{T}_{V}$. We'll show that $W U \in \mathcal{T}_{V}+\mathcal{K}_{V}$, and this will show multiplicative closure of $\mathcal{T}_{V}+\mathcal{K}_{V}$, since $\mathcal{K}_{V}$ is an ideal in $\mathcal{A}$. Without loss of generality, we may suppose that $W=V^{* m} W_{1}$ and $U=V^{* n} U_{1}$, for some elements $W_{1}$ and $U_{1}$ in $\mathcal{T}_{V}^{a}$ and some positive integers $n$ and $m$. Then $W U=$ $V^{* m+n} W_{1} U_{1}+V^{* m}\left[W_{1}, V^{* n}\right] U_{1}$ belongs to $\mathcal{T}_{V}+\mathcal{K}_{V}$, since $V^{* m+n} W_{1} U_{1} \in \mathcal{T}_{V}$ and $\left[W_{1}, V^{* n}\right] \in \mathcal{K}_{V}$.

Note that if $V$ is a pivotal isometry in the $\mathrm{C}^{*}$-algebra $\mathcal{A}$ and $\pi: \mathcal{A} \rightarrow C$ is a surjective $*$-homomorphism onto a $\mathrm{C}^{*}$-algebra $\mathcal{C}$ with kernel $\mathcal{K}_{V}$, then the elements $\pi\left(V^{* n} W\right)$ are dense in $\mathcal{C}$, where $n \geq 0$ and $W \in \mathcal{T}_{V}^{a}$, since $\pi\left(\mathcal{T}_{V}\right)=\mathcal{C}$.

For the index theory developed below we need to introduce a stronger condition on the isometry $V$ than being pivotal. However, although stronger, it is not very restrictive - we shall see a vast range of examples where the condition is satisfied in the next section.

Before introducing our condition we motivate it by considering the classical case of Toeplitz operators $T_{f}$ on $H^{2}$. If $f \in L^{\infty}$, it is an easy calculation to show that $f$ is a polynomial (in $\mathbf{z}$ ) if, and only if, there exists a nonnegative integer $N$ such that $T_{f}$ and $T_{\bar{f} \mathbf{z}^{N}}$ are analytic Toeplitz operators; equivalently, $T_{f}$ and $T_{f}^{*} \mathbf{S}^{N}$ commute with $\mathbf{S}$. One can then view $\mathcal{A}(\mathbf{T})$ as the $\mathrm{C}^{*}$-subalgebra of $\mathcal{B}\left(H^{2}\right)$ generated by the elements $T_{f}$ satisfying this condition.

Let $V$ be a proper isometry in a unital $\mathrm{C}^{*}$-algebra $\mathcal{A}$. We denote by $\mathcal{P}_{V}$ the set of all elements $W \in \mathcal{A}$ such that there exists $N \geq 0$ for which $W$ and $W^{*} V^{N}$ commute with $V$. It is immediate that in fact $W^{*} V^{N}$ also belongs to $\mathcal{P}_{V}$. Moreover, $\mathcal{P}_{V}$ is a unital subalgebra of $\mathcal{A}$.

We say $V$ is an essential isometry in $\mathcal{A}$ if $\mathcal{P}_{V}$ generates $\mathcal{A}$ as a $\mathrm{C}^{*}$-algebra.

We shall show below (Corollary 4.10) that for every unital $\mathrm{C}^{*}$-algebra $\mathcal{B}$, the element $\mathbf{S} \otimes 1$ is an essential isometry in $\mathcal{A}(\mathcal{B})=\mathcal{A}(\mathbf{C}) \otimes \mathcal{B}$.

Theorem 4.7. Let $V$ be an essential isometry in a unital $C^{*}$-algebra $\mathcal{A}$. Then $V$ is a pivotal isometry.

Proof. First note that since $\mathcal{P}_{V}$ generates $\mathcal{A}$ and the adjoint of an element in $\mathcal{P}_{V}$ is of the form $V^{* n} W$, where $W \in \mathcal{P}_{V}$ and $n \geq 0$, it follows that $\mathcal{A}$ is the closed linear span of products of elements of the above form $V^{* n} W$. Hence, by an argument 
along the lines given in the proof of Theorem 4.6, $\left(1-V^{n} V^{* n}\right)_{n}$ is an approximate unit for $\mathcal{K}_{V}$.

Now let $\mathcal{C}=\mathcal{A} / \mathcal{K}_{V}$ and $\pi: \mathcal{A} \rightarrow \mathcal{C}$ be the quotient homomorphism. Since $\mathcal{A}$ is the closed linear span of products of elements of the form $V^{* n} W$, where $W \in \mathcal{P}_{V}$ and $n \geq 0$, and since $\pi(V)$ is central in $\mathcal{C}$ and the elements $\pi\left(V^{* n} W\right)$ form a subalgebra of $\mathcal{C}$, it follows that these elements are dense in $\mathcal{C}$. Hence, if $W \in \mathcal{T}_{V}$, then $\pi(W)=\lim _{n \rightarrow \infty} \pi\left(V^{* m_{n}} W_{n}\right)$, for some sequence of elements $W_{n} \in \mathcal{P}_{V}$ and positive integers $m_{n}$, and therefore, by Theorem 4.4.

$$
\lim _{n}\left\|W-V^{* m_{n}} W_{n}\right\|=\lim _{n}\left\|\pi(W)-\pi\left(V^{* m_{n}} W_{n}\right)\right\|=0 .
$$

Hence, $V$ is a pivotal isometry in $\mathcal{A}$.

There is an obvious question that arises from the preceding result: Is a pivotal isometry necessarily essential? The answer is no, as the following example shows.

Example 4.8. The $\mathrm{C}^{*}$-algebra $\mathrm{QC}(\mathbf{T})$ of quasicontinuous functions on $\mathbf{T}$ is the intersection of the algebra $H^{\infty}+C(\mathbf{T})$ and the set of complex conjugates $\bar{f}$ of the functions $f$ in $H^{\infty}+C(\mathbf{T})$ 8, p. 176]. Note that $\mathrm{QC}(\mathbf{T})$ properly contains $C(\mathbf{T})$. Let $\mathcal{A}$ be the $\mathrm{C}^{*}$-subalgebra of $\mathcal{B}\left(H^{2}\right)$ generated by all Toeplitz operators $T_{f}$, where $f \in \mathrm{QC}(\mathbf{T})$. Clearly, the proper isometry $V=\mathbf{S}=T_{\mathbf{z}}$ belongs to $\mathcal{A}$, and $\mathcal{T}_{V}$ generates $\mathcal{A}$. The map

$$
\mathrm{QC}(\mathbf{T}) \rightarrow \mathcal{A} / \mathcal{K}\left(H^{2}\right), f \mapsto T_{f}+\mathcal{K}\left(H^{2}\right)
$$

is a $*$-isomorphism, by [8, Theorem 7.29]. Hence, if $W \in \mathcal{T}_{V}$, then $\mathbf{S}^{*} W \mathbf{S}=W$ implies $W=T_{f}$, for some element $f \in L^{\infty}$, and the existence of the $*$-isomorphism implies $f \in \mathrm{QC}(\mathbf{T})$. Hence, $f=h+k$, with $h \in H^{\infty}$ and $\bar{h} \in H^{\infty}+C(\mathbf{T})$ and $k \in C(\mathbf{T})$. Therefore, $T_{h}, T_{k} \in \mathcal{A}$ and since $k=\lim _{n} \overline{\mathbf{z}}^{m_{n}} k_{n}$, where $m_{n}$ are some positive integers and $k_{n}$ are some polynomials in $\mathbf{z}$, so $T_{f}=\lim _{n} V^{* m_{n}} W_{n}$, where $W_{n}=T_{\mathbf{z}^{m_{n} h+k_{n}}} \in \mathcal{T}_{V}^{a}$. Hence, $V$ is a pivotal isometry in $\mathcal{A}$.

On the other hand, since there is an element $f \in \mathrm{QC}(\mathbf{T})$ not contained in $C(\mathbf{T})$, the operator $T_{f} \in \mathcal{A}$ does not belong to $\mathcal{A}(\mathbf{T})$ (if $T_{f} \in \mathcal{A}(\mathbf{T})$, then $T_{f}=T_{h}+K$, for some function $h \in C(\mathbf{T})$ and some operator $K \in \mathcal{K}\left(H^{2}\right)$; then $T_{f-h} \in \mathcal{K}\left(H^{2}\right)$, from which it follows that $T_{f-h}=0$ and therefore $f=h$, contradicting the fact that $f \notin C(\mathbf{T}))$. Since $\mathcal{P}_{V}$ generates $\mathcal{A}(\mathbf{T})$, this shows that $V$ is not an essential isometry in $\mathcal{A}$.

Theorem 4.9. Let $\mathcal{B}$ be a unital $C^{*}$-algebra and let $V$ denote the proper isometry $\mathbf{S} \otimes 1$ in the $C^{*}$-algebra $\mathcal{A}=\mathcal{L}\left(H_{\mathcal{B}}^{2}\right)$. Then $\mathcal{P}_{V}$ is the set of all elements $W=$ $\sum_{n=0}^{N} \mathbf{S}^{n} \otimes b_{n}$, for $N \geq 0$ and elements $b_{n} \in \mathcal{B}$.

Proof. We regard $\mathcal{B}$ as a unital $\mathrm{C}^{*}$-subalgebra of $\mathcal{B}(L)$, for some Hilbert space $L$ and fix an orthonormal basis $\left(\eta_{i}\right)_{i}$ for $L$. We can also regard the algebra $\mathcal{A}=$ $M\left(\mathcal{K}\left(H^{2} \otimes \mathcal{B}\right)\right)$ as a unital $\mathrm{C}^{*}$-subalgebra of $\mathcal{B}\left(H^{2} \otimes L\right)$. Each bounded linear operator $W$ on $H^{2} \otimes L$ has an operator matrix $\left(W_{i j}\right)_{i j}$, with entries $W_{i j} \in \mathcal{B}\left(H^{2}\right)$, given by

$$
\left\langle g \mid W_{i j} f\right\rangle=\left\langle g \otimes \eta_{i} \mid W\left(f \otimes \eta_{j}\right)\right\rangle \quad\left(f, g \in H^{2}\right)
$$

Clearly, $W$ commutes with $V=\mathbf{S} \otimes 1$ if, and only if, each matrix entry $W_{i j}$ commutes with $\mathbf{S}$. In this case, $W_{i j}$ is an analytic Toeplitz operator, so $W_{i j}=T_{f_{i j}}$, for some function $f_{i j}$ belonging to the Hardy space $H^{\infty}$ [9, p. 79].

Now suppose that $W$ is an element of $\mathcal{P}_{V}$; that is, $W$ is an element of $\mathcal{A}$ such that $W$ and $W^{*} V^{N}$ commute with $V$, for some positive integer $N$. It follows from 
the preceding paragraph that the operator matrices of $W$ and $W^{*} V^{N}$ are of the form $\left(T_{f_{i j}}\right)$ and $\left(T_{g_{i j}}\right)$, respectively, where $f_{i j}$ and $g_{i j}$ belong to $H^{\infty}$. Clearly, the operator $W^{*} V^{N}$ also has an operator matrix of the form $\left(T_{\bar{f}_{j i} \mathbf{z}^{N}}\right)_{i j}$. Therefore, $\bar{f}_{j i} \mathbf{z}^{N}=g_{i j}$, for all indices $i$ and $j$. It follows that $f_{i j}$ belongs to the linear span in $H^{\infty}$ of the vectors $1, \mathbf{z}, \ldots, \mathbf{z}^{N}$ and therefore,

$$
T_{f_{i j}}=\sum_{m=0}^{N} \lambda_{i j}(m) \mathbf{S}^{m},
$$

for scalars $\lambda_{i j}(m)$.

Let $E=1-\mathbf{S S}^{*}$. The operator matrix $\left(\lambda_{i j}(m) E\right)_{i j}$ is the matrix of the operator $T_{m}=\left(E \mathbf{S}^{* m} \otimes 1\right) W(E \otimes 1)$, for $m=0, \ldots, N$. The corresponding scalar matrix $\left(\lambda_{i j}(m)\right)_{i j}$ is the matrix of a norm-bounded operator, $b_{m}$ say, on $L$. (The sesquilinear form

$$
\left(\eta, \eta^{\prime}\right) \mapsto\left\langle e_{0} \otimes \eta \mid T_{m}\left(e_{0} \otimes \eta^{\prime}\right)\right\rangle
$$

defines a norm-bounded linear operator $b_{m}$ on $L$ with matrix $\left(\lambda_{i j}(m)\right)_{i j}$.) From equation (7) we now get

$$
W=\sum_{m=0}^{N} \mathbf{S}^{m} \otimes b_{m} .
$$

Since, for $m=0, \ldots, N,\left(E \mathbf{S}^{* m} \otimes 1\right) W(E \otimes 1)=E \otimes b_{m}$ belongs to $\mathcal{A}$, and therefore to $(E \otimes 1) M\left(\mathcal{K}\left(H^{2}\right) \otimes \mathcal{B}\right)(E \otimes 1)=E \otimes \mathcal{B}$, it follows that $b_{m} \in \mathcal{B}$.

Corollary 4.10. The proper isometry $V=\mathbf{S} \otimes 1$ is an essential isometry of the $C^{*}$-algebra $\mathcal{A}(\mathcal{B})=\mathcal{A}(\mathbf{C}) \otimes \mathcal{B}$.

The next result shows that for an essential isometry, the corresponding Toeplitz elements can be represented by $\mathcal{B}$-Toeplitz operators with continuous symbols, thereby opening up the index theory of these Toeplitz elements for analysis.

Theorem 4.11. Let $V$ be an essential proper isometry in a unital $C^{*}$-algebra $\mathcal{A}$ and let $\pi: \mathcal{A} \rightarrow \mathcal{C}$ be a $*$-homomorphism onto a unital $C^{*}$-algebra $\mathcal{C}$ with kernel $\mathcal{K}_{V}$. Let $\psi$ be the representation on $H_{\mathcal{B}_{V}}^{2}$ associated to $V$. Then $\psi(\mathcal{A}) \subseteq \mathcal{A}\left(\mathcal{B}_{V}\right)$ and there exists a unique $*$-homomorphism $\rho: \mathcal{C} \rightarrow C^{*}\left(M_{\mathbf{z}}\right) \otimes \mathcal{B}_{V}$ such that we have a commutative diagram

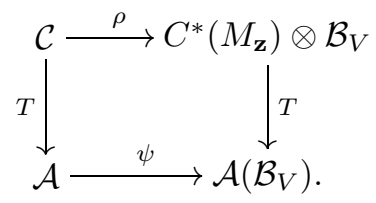

If $\mathcal{K}_{V}$ is essential as an ideal in $\mathcal{A}$, then $\rho$ is isometric.

Proof. First we show $\psi(\mathcal{A}) \subseteq \mathcal{A}\left(\mathcal{B}_{V}\right)$. Since $\mathcal{P}_{V}$ generates $\mathcal{A}$, because $V$ is essential in $\mathcal{A}$, it suffices to show that $\psi\left(\mathcal{P}_{V}\right) \subseteq \mathcal{A}\left(\mathcal{B}_{V}\right)$. However, if $W \in \mathcal{P}_{V}$, it is clear that $\psi(W)$ belongs to the subset $\mathcal{P}_{\mathbf{S} \otimes 1}$ of $\mathcal{L}\left(H_{\mathcal{B}_{V}}^{2}\right)$. Hence, by Theorem 4.9, $\psi(W)=$ $\sum_{n=0}^{N} \mathbf{S}^{n} \otimes b_{n}$, for some elements $b_{0}, \ldots, b_{N}$. Therefore, $\psi(W)$ belongs to $\mathcal{A}(\mathbf{T}) \otimes$ $\mathcal{B}_{V}=\mathcal{A}\left(\mathcal{B}_{V}\right)$, as required. 
Uniqueness of $\rho$ is immediate, so we turn to showing existence. Again suppose $W \in \mathcal{P}_{V}$, so that $\psi(W)=\sum_{n=0}^{N} \mathbf{S}^{n} \otimes b_{n}$. Then

$$
T_{\sum_{n=0}^{N} M_{\mathbf{z}^{n}} \otimes b_{n}}=\sum_{n=0}^{N} \mathbf{S}^{n} \otimes b_{n}=\psi(W),
$$

so that $W \in \mathcal{P}_{V}$ implies $\psi(W)=T_{\tilde{W}}$, for some element $\tilde{W} \in C^{*}\left(M_{\mathbf{z}}\right) \otimes \mathcal{B}_{V}$. It follows immediately that a similar statement is true for all elements of $\mathcal{T}_{V}$ of the form $V^{* n} W$, where $n \geq 0$ and $W \in \mathcal{P}_{V}$ and since $\mathcal{T}_{V}$ is the norm closure of such elements, one can now use the fact that the map

$$
C^{*}\left(M_{\mathbf{z}}\right) \otimes \mathcal{B}_{V}, \quad W \mapsto T_{W},
$$

is an isometry to deduce that for any element $W \in \mathcal{T}_{V}$, there is an element $\tilde{W}$ in $C^{*}\left(M_{\mathbf{z}}\right) \otimes \mathcal{B}_{V}$ such that $\psi(W)=T_{\tilde{W}}$.

Hence, if $f \in \mathcal{C}$, then there is a unique element $\rho(f)$ in $C^{*}\left(M_{\mathbf{z}}\right) \otimes \mathcal{B}_{V}$ such that $T_{\rho(f)}=\psi\left(T_{f}\right)$. It is clear that this defines a map $\rho: \mathcal{C} \rightarrow C^{*}\left(M_{\mathbf{z}}\right) \otimes \mathcal{B}_{V}$ making the diagram above commute, and it is trivially verified that $\rho$ is a $*$-linear map.

If $f \in \mathcal{C}$, then $\|\rho(f)\|=\left\|T_{\rho(f)}\right\|=\left\|\psi\left(T_{f}\right)\right\| \leq\left\|T_{f}\right\|=\|f\|$. Thus, $\rho$ is normdecreasing (and if $\mathcal{K}_{V}$ is essential in $\mathcal{A}$, then $\psi$ is isometric and therefore, clearly, so is $\rho$ ).

Let $W$ be an element of $\mathcal{A}$ commuting with $V$ and let $f \in \mathcal{C}$. Then $T_{\rho(f \pi(W))}=$ $\psi\left(T_{f \pi(W)}\right)=\psi\left(T_{f} W\right)=\psi\left(T_{f}\right) \psi(W)=T_{\rho(f)} T_{\rho \pi(W)}=T_{\rho(f) \rho \pi(W)}$, where the last equality follows from the fact that $\rho \pi(W)$ is an analytic-type element of $\mathcal{L}_{\mathcal{B}_{V}}$, since $T_{\rho \pi(W)}=\psi(W)$ commutes with $\mathbf{S} \otimes 1$. Thus, $\rho(f \pi(W))=\rho(f) \rho \pi(W)$.

Let $\Delta=\pi(V)$ and note that $\rho(\Delta)=\mathbf{U}$. Let $f_{j}=\Delta^{* N_{j}} \pi\left(W_{j}\right)$, for $j=1,2$, where $W_{1}$ and $W_{2}$ are elements of $\mathcal{A}$ commuting with $V$ and $N_{1}, N_{2} \geq 0$. Then, if $\tilde{W}_{j}=\pi\left(W_{j}\right)$

$$
\begin{gathered}
\rho\left(f_{1} f_{2}\right)=\rho\left(\left(\Delta^{N_{1}+N_{2}}\right)^{*} \tilde{W}_{1} \tilde{W}_{2}\right)=\rho\left(\left(\Delta^{N_{1}+N_{2}}\right)^{*}\right) \rho\left(\tilde{W}_{1} \tilde{W}_{2}\right) \\
=\rho\left(\Delta^{N_{1}+N_{2}}\right)^{*} \rho\left(\tilde{W}_{1} \tilde{W}_{2}\right)=\left(\mathbf{U}^{N_{1}+N_{2}}\right)^{*} \rho\left(\tilde{W}_{1}\right) \rho\left(\tilde{W}_{2}\right) \\
=\mathbf{U}^{* N_{1}} \rho\left(\tilde{W}_{1}\right) \mathbf{U}^{* N_{2}} \rho\left(\tilde{W}_{2}\right)=\rho\left(f_{1}\right) \rho\left(f_{2}\right) .
\end{gathered}
$$

Thus, $\rho$ is multiplicative on the elements of $\mathcal{C}$ of the form $f_{j}$ and therefore, since these elements are dense in $\mathcal{C}, \rho$ is multiplicative on all of $\mathcal{C}$.

We call the map $\rho$ of the preceding theorem the representation of $\mathcal{C}$ in $C^{*}\left(M_{\mathbf{z}}\right) \otimes$ $\mathcal{B}_{V}$ subordinate to the representation $\psi$ of $\mathcal{A}$.

Corollary 4.12. Let $\tau$ be a tracial state on $\mathcal{B}_{V}$ and $\operatorname{tr}_{\tau}$ the associated trace on $\mathcal{K}\left(H_{\mathcal{B}_{V}}^{2}\right)$. If $f \in \operatorname{Inv} \mathcal{C}$, the $\mathcal{B}_{V}$-Toeplitz operator $\psi\left(T_{f}\right)$ is a $\operatorname{tr}_{\tau}$-Fredholm operator and

$$
\operatorname{index}_{\tau} \psi\left(T_{f}\right)=-\omega_{\tau} \rho(f),
$$

where $\omega_{\tau}$ is the topological index on $C\left(\mathbf{T}, \mathcal{B}_{V}\right)=C^{*}\left(M_{\mathbf{z}}\right) \otimes \mathcal{B}_{V}$ associated to $\tau$.

If $\mathcal{K}_{V}$ is essential as a closed ideal in $\mathcal{A}$, and $f \in \mathcal{C}$, then $f$ is invertible if $\psi\left(T_{f}\right)$ is a $\operatorname{tr}_{\tau}$-Fredholm operator.

Proof. Let $f \in \mathcal{C}$. Since $\psi\left(T_{f}\right)=T_{\rho(f)}$, Theorem 3.3 implies that $\psi\left(T_{\varphi}\right)$ is $\operatorname{tr}_{\tau^{-}}$ Fredholm if, and only if, $\rho(f)$ is invertible in $C^{*}\left(M_{\mathbf{z}}\right) \otimes \mathcal{B}_{V}$. Hence, if $f \in \operatorname{Inv} \mathcal{C}$, $\rho(f)$ is invertible and, again by Theorem 3.3. Equation (9) holds. 
If $\mathcal{K}_{V}$ is essential as a closed ideal in $\mathcal{A}$, and $f \in \mathcal{C}$, then $f$ is invertible if $\psi\left(T_{f}\right)$ is a $\operatorname{tr}_{\tau}$-Fredholm operator, since $\rho(f)$ is invertible and the $*$-homomorphism $\rho: \mathcal{C} \rightarrow C^{*}\left(M_{\mathbf{z}}\right) \otimes \mathcal{B}_{V}$ is injective in this case.

\section{Essential isometries in Classes of $\mathrm{C}^{*}$-ALGebras}

In this section we shall give examples of essential isometries in a wide variety of $\mathrm{C}^{*}$-algebras to illustrate the range of the theory developed so far.

Example 5.1. Consider the universal $\mathrm{C}^{*}$-algebra $\mathcal{A}=C^{*}\left(U_{1}, U_{2}\right)$ generated by two commuting isometries $U_{1}$ and $U_{2}$. This algebra has the property that if $\mathcal{D}$ is any unital $\mathrm{C}^{*}$-algebra and $V_{1}$ and $V_{2}$ are commuting isometries in $\mathcal{D}$, then there is a unique $*$-homomorphism $\pi: \mathcal{A} \rightarrow \mathcal{D}$ such that $\pi\left(U_{1}\right)=V_{1}$ and $\pi\left(U_{2}\right)=$ $V_{2}$. Perhaps surprisingly, $\mathcal{A}$ is not nuclear, nor even subnuclear; see 18 , where a detailed investigation is made of this algebra. The proper isometry $V=U_{1} U_{2}$ is essential in $\mathcal{A}$, since $U_{1}$ and $U_{2}$ clearly belong to $\mathcal{P}_{V}$ and generate $\mathcal{A}$. Since $U_{1}+\mathcal{K}_{V}$ and $U_{2}+\mathcal{K}_{V}$ are commuting isometries in $\mathcal{C}=\mathcal{A} / \mathcal{K}_{V}$ with product the unitary $V+\mathcal{K}_{V}$, both $U_{1}+\mathcal{K}_{V}$ and $U_{2}+\mathcal{K}_{V}$ are themselves unitaries and therefore, since $\mathcal{C}$ is generated by them, it is commutative. Thus, $\mathcal{K}_{V}$ is equal to the closed commutator ideal of $\mathcal{A}$.

The $\mathrm{C}^{*}$-algebra $\mathcal{B}_{V}$ can be calculated explicitly in this case. In [18] it is shown that $\mathcal{B}_{V}=C^{*}\left(\mathbf{Z} * \mathbf{Z}_{2}\right)$, the full $\mathrm{C}^{*}$-algebra on the group free product of the group $\mathbf{Z}$ and the two-element group $\mathbf{Z}_{2}$.

One should note that it is exceptional that we can calculate $\mathcal{B}_{V}$ as explicitly as this - in general, it appears to be a difficult problem to calculate this algebra. Despite this, we can use $\mathcal{B}_{V}$, via the representation theorem, Theorem 4.1, to great effect in the index theory, as we'll see in the next two sections.

Example 5.2. Similar considerations show that if $\mathcal{A}$ is any $\mathrm{C}^{*}$-algebra generated by $n$ commuting proper isometries $U_{1}, \ldots, U_{n}$, then $V=U_{1} \cdots U_{n}$ is an essential proper isometry for $\mathcal{A}$ and $1-V V^{*}$ generates the closed commutator ideal of $\mathcal{A}$.

Example 5.3. Let $\Gamma$ be an ordered group with dual group $G$. The Hardy Hilbert space of $\Gamma$ is defined to be the closed linear span $H^{2}(\Gamma)$ in $L^{2}(G)$ of all elements $\varepsilon_{x}$, where $x \in \Gamma^{+}$. Recall that $\varepsilon_{x}$ is the continuous function $g \mapsto g(x)$ on $G$. If $f \in$ $C(G)$, we define the Toeplitz operator $T_{f} \in \mathcal{B}\left(H^{2}(\Gamma)\right)$ to be the compression of the multiplication operator $M_{f} \in \mathcal{B}\left(L^{2}(G)\right)$ to $H^{2}(\Gamma)$. The Toeplitz algebra of $\Gamma$ is the $\mathrm{C}^{*}$-subalgebra $\mathcal{A}=\mathcal{A}(\Gamma)$ of $\mathcal{B}\left(H^{2}(\Gamma)\right)$ generated by all operators $T_{f}(f \in C(G))$.

These algebras have been extensively studied; see [11, 16, 18.

If $z$ is an order unit of $\Gamma$, the proper isometry $V=T_{\varepsilon_{z}}$ is essential for $\mathcal{A}=$ $\mathcal{A}(\Gamma)$, since the operators $V_{x}=T_{\varepsilon_{x}}$, for $x \in \Gamma^{+}$, are clearly contained in $\mathcal{P}_{V}$ and generate $\mathcal{A}$.

Note that $\mathcal{K}_{V}$ is equal to the closed commutator ideal $\mathcal{K}(\Gamma)$ of $\mathcal{A}$. For, if $0 \leq$ $x, y \in \Gamma$, then $V_{x+y} V_{x+y}^{*}=V_{x} V_{y} V_{y}^{*} V_{x}^{*} \leq V_{x} V_{x}^{*}$. Hence, $0 \leq x \leq y$ implies $1-$ $V_{x} V_{x}^{*} \leq 1-V_{y} V_{y}^{*}$. It follows that if $0 \leq x$, then $x \leq N z$, for some $N>0$, and therefore $1-V_{x} V_{x}^{*} \leq 1-V^{N} V^{* N}$, implying that $1-V_{x} V_{x}^{*} \in \mathcal{K}_{V}$. Hence, since the elements $1-V_{x} V_{x}^{*}$ clearly generate $\mathcal{K}(\Gamma)$ as a closed ideal, $\mathcal{K}(\Gamma) \subseteq \mathcal{K}_{V}$. The reverse containment is trivial, so $\mathcal{K}_{V}=\mathcal{K}(\Gamma)$.

Note that there is a unique $*$-homomorphism $\pi$ from $\mathcal{A}$ onto $C(G)$ such that $\pi\left(T_{\varepsilon_{x}}\right)=\varepsilon_{x}$, for all $x \in \Gamma$ and that $\operatorname{ker}(\pi)=\mathcal{K}_{V}$; see [11]. It follows easily that the map $T: f \mapsto T_{f}$ is the $V$-related right inverse for $\pi$ constructed in Theorem 4.4. 
In particular, the set $\mathcal{T}_{V}$ of $V$-Toeplitz operators is equal to the set of all Toeplitz operators $T_{f}$ on $H^{2}(\Gamma)$ with continuous symbol $f$ in $C(G)$.

See [1] and [16] for more details on Toeplitz algebras of ordered groups.

Example 5.4. Let $N$ be a positive integer and $D$ a symmetric ball in $\mathbf{C}^{N}$, for some norm on $\mathbf{C}^{N}$. Let $S$ be the Shilov boundary of $D$ and $m$ the Bott-Korányi measure on $S$. Let $P_{0}(S)$ be the algebra of all polynomials on $S$. The closure of $P_{0}(S)$ in $L^{2}(S)=L^{2}(m)$ is denoted by $H^{2}(D)$ and called the Hardy Hilbert space of the ball $D$. If $f \in C(S)$, we define the Toeplitz operator $T_{f} \in \mathcal{B}\left(H^{2}(D)\right)$ to be the compression of the multiplication operator $M_{f}$ on $L^{2}(S)$ to $H^{2}(D)$.

The Toeplitz algebra $\mathcal{A}(D)$ of $D$ is the $\mathrm{C}^{*}$-subalgebra of $\mathcal{B}\left(H^{2}(D)\right)$ generated by all Toeplitz operators $T_{f}(f \in C(S))$. It is a Type I $\mathrm{C}^{*}$-algebra 24].

Suppose now the symmetric ball $D$ is of tube type. In this case, as we observed earlier, its Shilov boundary $S$ carries a polynomial $\Delta$ (its Koecher norm function) that is of modulus one on $S$ and for which we have $\bar{z}_{j} \Delta$ is a polynomial, for $j=1, \ldots, n$, where, of course, $z_{j}$ denotes the $j$ th coordinate function on $\mathbf{C}^{n}$ restricted to $S$. The proper isometry $V=T_{\Delta}$ is essential for $\mathcal{A}(D)$, since the Toeplitz operators $T_{z_{j}}$ belong to $\mathcal{P}_{V}$ and generate $\mathcal{A}(D)$.

In this case, $\mathcal{K}_{V}$ is equal to the closed commutator ideal $\mathcal{K}(D)$ of $\mathcal{A}(D)$. To see this, note that it suffices to show $\mathcal{A}(D) / \mathcal{K}_{V}$ is commutative. Since the operators $T_{p}+\mathcal{K}_{V}\left(p \in P_{0}(S)\right)$ generate $\mathcal{A}(D) / \mathcal{K}_{V}$, and commute with each other, it suffices to show each element $T_{p}+\mathcal{K}_{V}$ is normal. To see this, first observe that there is some positive integer $N$ such that $\bar{p} \Delta^{N} \in P_{0}(S)$. Hence, $T_{p} T_{\bar{p}} T_{\Delta^{N}}=T_{p} T_{\bar{p} \Delta^{N}}=$ $T_{\bar{p} \Delta^{N}} T_{p}=T_{\bar{p}} T_{p} T_{\Delta^{N}}$. Since $T_{\Delta}+\mathcal{K}_{V}$ is a unitary in $\mathcal{A}(D) / \mathcal{K}_{V}$, we conclude that $T_{p} T_{\bar{p}}+\mathcal{K}_{V}=T_{\bar{p}} T_{p}+\mathcal{K}_{V}$.

It is known that there is a unique $*$-homomorphism $\pi: \mathcal{A}(D) \rightarrow C(S)$ such that $\pi\left(T_{f}\right)=f$, for all $f \in C(S)$, and that $\operatorname{ker} \pi=\mathcal{K}(D)$; see [19] for a quick proof. It follows easily that the map $T: f \mapsto T_{f}$ is the $V$-related right inverse for $\pi$ constructed in Theorem 4.4 and that the set $\mathcal{T}_{V}$ of $V$-Toeplitz operators is equal to the set of all Toeplitz operators $T_{f}$ on $H^{2}(D)$ with continuous symbol $f$ in $C(S)$.

Toeplitz operators over the Hardy spaces of symmetric balls have been extensively studied, especially by H. Upmeier. His book [26] should be consulted for the theory of Toeplitz operators in this context.

\section{INDEX THEORY FOR TOEPLITZ OPERATORS ON HARDY SPACES OF ORDERED GROUPS}

Let $\Gamma$ be an ordered group. The algebra $\mathcal{A}(\Gamma)$ has a universal property [11, Theorem 1.3] that enables us to deduce that there is a homomorphism

$$
\alpha: G \rightarrow \operatorname{Aut} \mathcal{A}(\Gamma), \quad g \mapsto \alpha_{g},
$$

on the dual group $G=\hat{\Gamma}$ uniquely determined by the condition

$$
\alpha_{g}\left(T_{\varepsilon_{x}}\right)=g(x) T_{\varepsilon_{x}} \quad\left(x \in \Gamma^{+}, g \in G\right) .
$$

This action is continuous in the sense that for all $W \in \mathcal{A}(\Gamma)$, the map

$$
G \rightarrow \mathcal{A}(\Gamma), \quad g \mapsto \alpha_{g}(W),
$$


is continuous. This enables us to define a norm-decreasing positive linear map $E: \mathcal{A}(\Gamma) \rightarrow \mathcal{A}(\Gamma)$ by setting

$$
E(W)=\int \alpha_{g}(W) \mathrm{d} g,
$$

where $\mathrm{d} g$ denotes the normalised Haar measure on $G$. We call $E$ the mean on $\mathcal{A}(\Gamma)$ associated to the natural action $\alpha$.

If $z$ is an order unit of $\Gamma$, then $V=T_{\varepsilon_{z}}$ is an essential isometry of $\mathcal{A}(\Gamma)$, as we observed in Example 5.3. Clearly, $\alpha_{g}\left(Q_{V}\right)=Q_{V}$, for all $g \in G$. Thus, we have an induced continuous natural action

$$
G \rightarrow \operatorname{Aut} \mathcal{B}_{V},\left.\quad g \mapsto \alpha_{g}\right|_{\mathcal{B}_{V}},
$$

of $G$ on $\mathcal{B}_{V}$. Note also that $E\left(Q_{V}\right)=Q_{V}$ and $E\left(\mathcal{B}_{V}\right) \subseteq \mathcal{B}_{V}$.

These observations will be used at several points in the sequel.

Our aim is to prove an index theorem for Toeplitz operators with continuous symbol on the Hardy space associated to an ordered group $\Gamma$. Before doing this we make an observation that justifies the hypothesis we adopt in the theorem: If one is going to prove a nontrivial index theorem for the Toeplitz operators on $H^{2}(\Gamma)$ relative to a densely-defined lower semicontinuous trace $\operatorname{tr}$ on $\mathcal{K}(\Gamma)$, the closed commutator ideal of $\mathcal{A}(\Gamma)$, then $\Gamma$ will have to admit an order unit; equivalently, it will have to contain a largest proper ideal. For, the map

$$
\Gamma \rightarrow \mathbf{R}, \quad x \mapsto-\text { index }_{\operatorname{tr}}\left(T_{\varepsilon_{x}}\right)=\operatorname{tr}\left(T_{\varepsilon_{x}}^{*} T_{\varepsilon_{x}}-T_{\varepsilon_{x}} T_{\varepsilon_{x}}^{*}\right)
$$

will be a nonzero positive homomorphism and its kernel is then the largest proper ideal of $\Gamma$. This justifies our assumption that $\Gamma$ admits an order unit in the index theorem we prove below (Theorem 6.3).

Lemma 6.1. Let $\Gamma$ be an ordered group admitting an order unit $z$ and let $\varphi: \Gamma \rightarrow \mathbf{R}$ be the unique positive homomorphism such that $\varphi(z)=1$. If $[0, z]$ is the set of all elements $x$ in $\Gamma$ such that $0 \leq x \leq z$ and $\tau:[0, z] \rightarrow \mathbf{R}^{+}$is a function such that $\tau(z)=1$ and $\tau(x+y)=\tau(x)+\tau(y)$, for all $x, y \in[0, z]$ for which $x+y \in[0, z]$, then $\tau(x)=\varphi(x)$, for all $x \in[0, z]$.

Proof. Suppose $\tau:[0, z] \rightarrow \mathbf{R}^{+}$has the additivity property stated in the hypothesis. Extend $\tau$ to a function $\tau_{0}: \Gamma \rightarrow \mathbf{R}$ by setting $\tau_{0}(x)=N+\tau(x-N z)$, for $x$ in $\Gamma$, where $N$ is the greatest integer such that $N z \leq x$. An elementary calculation shows that $\tau_{0}$ is a positive homomorphism. Since $\tau_{0}(z)=1$, we must have $\tau_{0}=\varphi$.

Theorem 6.2. Let $\Gamma$ be an ordered group with an order unit $z$ and let $V$ be the essential isometry $T_{\varepsilon_{z}}$ in the Toeplitz algebra $\mathcal{A}(\Gamma)$. Then there is a unique tracial state $\tau$ on $\mathcal{B}_{V}$ that is invariant for the natural action of the dual group $G$ of $\Gamma$ on $\mathcal{B}_{V}$.

Proof. For notational convenience we'll write $Q$ for $Q_{V}$ in this proof.

If $x \in \Gamma$, set $V_{x}=T_{\varepsilon_{x}}$ and if $x \in \Gamma^{+}$, set $P_{x}=1-V_{x} V_{x}^{*}$. Then $\mathcal{A}(\Gamma)$ is the closed linear span of the elements of the form $V_{x} V_{y}^{*}$, where $x, y \in \Gamma^{+}$and $\mathcal{K}_{V}$ is the closure of the linear span $\mathcal{K}_{0}$ of the elements $P_{x} V_{y}$, where $x \in \Gamma^{+}$and $y \in \Gamma$ [14] (recall that $\mathcal{K}_{V}$ is equal to the closed commutator ideal of $\mathcal{A}(\Gamma)$, as we observed earlier). Now $E\left(P_{x} V_{y}\right)=\int g(y) P_{x} V_{y} \mathrm{~d} g=\delta_{0, y} P_{x}$, so if $K_{0}$ is the linear span of the projections $P_{x}$, and $K$ is the closure of $K_{0}$, then $E\left(\mathcal{K}_{0}\right)=K_{0}$ and $E\left(\mathcal{K}_{V}\right)=K$. Note that if $x, y \in \Gamma^{+}$, then $P_{x} P_{y}=P_{x \wedge y}$, where $x \wedge y$ is the minimum of $x$ and $y$. 
Hence, $K$ is a commutative $\mathrm{C}^{*}$-subalgebra of $\mathcal{A}(\Gamma)$. Since $\mathcal{B}_{V}=Q \mathcal{K}_{V} Q$, we have $E\left(\mathcal{B}_{V}\right)=Q K Q=Q K$; clearly, $Q K$ is the closed linear span of all the projections $P_{x}$, where $0 \leq x \leq z$.

Suppose now $\tau_{1}, \tau_{2}: \mathcal{B}_{V} \rightarrow \mathbf{C}$ are invariant tracial states on $\mathcal{B}_{V}$ (that is, they are invariant for the natural action of $G$ on $\left.\mathcal{B}_{V}\right)$. If $W \in \mathcal{B}_{V}$, then $\tau_{j} E(W)=$ $\int \tau_{j} \alpha_{g}(W) \mathrm{d} g=\int \tau_{j}(W) \mathrm{d} g=\tau_{j}(W)$. Hence, to show $\tau_{1}$ and $\tau_{2}$ are equal we need only show they are equal on $Q K$. Therefore, we need only show $\tau_{1}\left(P_{x}\right)=\tau_{2}\left(P_{x}\right)$, for all $x$ such that $0 \leq x \leq z$. Let $x, y \in \Gamma^{+}$and suppose $x+y \leq z$. Since, as is readily verified, $Q V_{x} Q=Q V_{x}$ and $V_{x}^{*} Q V_{x}=P_{z-x}$, we have

$$
\begin{gathered}
\tau_{j}\left(P_{x+y}\right)=\tau_{j}\left(Q\left(1-V_{x} V_{y} V_{y}^{*} V_{x}^{*}\right) Q\right)=\tau_{j}\left(Q\left(1-V_{x}\left(1-P_{y}\right) V_{x}^{*}\right) Q\right) \\
=\tau_{j}\left(Q P_{x} Q\right)+\tau_{j}\left(Q V_{x} P_{y} V_{x}^{*} Q\right)=\tau_{j}\left(P_{x}\right)+\tau_{j}\left(Q V_{x} Q P_{y} Q V_{x}^{*} Q\right) \\
=\tau_{j}\left(P_{x}\right)+\tau_{j}\left(Q V_{x}^{*} Q Q V_{x} Q P_{y}\right)=\tau_{j}\left(P_{x}\right)+\tau_{j}\left(P_{z-x} P_{y}\right) \\
=\tau_{j}\left(P_{x}\right)+\tau_{j}\left(P_{(z-x) \wedge y}\right)=\tau_{j}\left(P_{x}\right)+\tau_{j}\left(P_{y}\right) .
\end{gathered}
$$

If $\varphi: \Gamma \rightarrow \mathbf{R}$ is the unique positive homomorphism such that $\varphi(z)=1$, then by Lemma 6.1] $\tau_{j}\left(P_{x}\right)=\varphi(x)$, for all $x$ in $\Gamma$ such that $0 \leq x \leq z$. Hence, $\tau_{1}=\tau_{2}$, as required.

We turn now to showing the existence of an invariant tracial state on $\mathcal{B}_{V}$. To begin, suppose that $W=\sum_{i=1}^{M} \lambda_{i} Q_{i}$, where $Q_{i}=P_{x_{i}}$, with $x_{i} \in \Gamma$ and $0 \leq x_{i} \leq z$ and $\lambda_{i} \in \mathbf{C}$. We claim that $\left|\sum_{i=1}^{M} \lambda_{i} \varphi\left(x_{i}\right)\right| \leq\|W\|$. To show this we may clearly suppose that $Q_{1} \geq Q_{2} \geq \cdots \geq Q_{M}$ by supposing that $x_{1} \geq x_{2} \geq \cdots \geq x_{M}$. For notational convenience set $x_{M+1}=0$ and $Q_{M+1}=P_{x_{M+1}}=0$. Also, set $\mu_{i}=\lambda_{1}+\lambda_{2}+\cdots+\lambda_{i}$, for $i=1,2, \ldots, M$. Then

$$
W=\sum_{i=1}^{M} \mu_{i}\left(Q_{i}-Q_{i+1}\right)
$$

and therefore, since the projections $Q_{1}-Q_{2}, \ldots, Q_{M}-Q_{M+1}$ are pairwise orthogonal, we have $\|W\|=\max \left\{\left|\mu_{i}\right| \mid Q_{i}-Q_{i+1} \neq 0\right\}$. Hence,

$$
\begin{aligned}
& \left|\sum_{i=1}^{M} \lambda_{i} \varphi\left(x_{i}\right)\right|=\left|\sum_{i=1}^{M} \mu_{i} \varphi\left(x_{i}-x_{i+1}\right)\right| \leq \sum_{i=1}^{M}\left|\mu_{i}\right| \varphi\left(x_{i}-x_{i+1}\right) \\
& \leq\|W\| \sum_{i=1}^{M} \varphi\left(x_{i}-x_{i+1}\right)=\|W\| \varphi\left(x_{1}\right) \leq\|W\| \varphi(z)=\|W\| .
\end{aligned}
$$

The norm estimate $\left|\sum_{i=1}^{M} \lambda_{i} \varphi\left(x_{i}\right)\right| \leq\|W\|$ shows that we can well-define a normdecreasing linear map $\tau_{0}$ on the linear span of the projections $P_{x}$, where $0 \leq x \leq z$, by setting $\tau_{0}(W)=\sum_{i=1}^{M} \lambda_{i} \varphi\left(x_{i}\right)$. This extends to a norm-decreasing linear map $\tau_{0}: Q K \rightarrow \mathbf{C}$. Since $\left\|\tau_{0}\right\| \leq 1$ and $\tau_{0}(Q)=\varphi(z)=1, \tau_{0}$ is a state on $Q K$.

Now we define $\tau: \mathcal{B}_{V} \rightarrow \mathbf{C}$ by setting $\tau=\tau_{0} \circ E$. Clearly, $\tau$ is an invariant state of $\mathcal{B}_{V}$. We claim now that $\tau$ is tracial and to see this we need only show that $\tau\left(W_{1} W_{2}-W_{2} W_{1}\right)=0$, for all elements $W_{1}$ and $W_{2}$ in $\mathcal{K}_{V}$ of the form $W_{1}=P_{x} V_{y} Q$ and $W_{2}=P_{r} V_{s} Q$, where $x, y, r, s \in \Gamma$ and $0 \leq x, r \leq z$, since the elements of this form have a closed linear span equal to $\mathcal{B}_{V}$. Let $W=W_{1} W_{2}-W_{2} W_{1}$. Then

$$
\begin{gathered}
E(W)=\int P_{x} g(y) V_{y} P_{r} g(s) V_{s} Q-P_{r} g(s) V_{s} P_{x} g(y) V_{y} Q \mathrm{~d} g \\
=\delta_{y,-s}\left(P_{x} V_{y} P_{r} V_{s} Q-P_{r} V_{s} P_{x} V_{y} Q\right),
\end{gathered}
$$


since $\int g(y+s) \mathrm{d} g=\delta_{y,-s}$. Clearly, we may suppose (to show $\tau_{0}(E(W))=0$ ) that $y=-s$ and therefore we restrict ourselves to commutators $W$ such that $E(W)$ is of the form

$$
E(W)=P_{x} V_{y} P_{r} V_{y}^{*} Q-P_{r} V_{y}^{*} P_{x} V_{y} Q .
$$

Replacing $W$ by $-W$ if necessary, we may also suppose that $y \geq 0$. In this case, $V_{y} P_{r} V_{y}^{*}=P_{r+y}-P_{y}$; also, $V_{y}^{*} P_{x} V_{y}=0$ if $y>x$ and $V_{y}^{*} P_{x} V_{y}=P_{x-y}$ if $y \leq x$. Hence, in the case that $y>x$, we have

$$
\tau_{0} E(W)=\tau_{0}\left(P_{x}\left(P_{r+y}-P_{y}\right)\right)=\tau_{0}\left(P_{x \wedge(r+y)}-P_{x \wedge y}\right)=\tau_{0}\left(P_{x}-P_{x}\right)=0 .
$$

In the case that $y \leq x$, we have

$$
\begin{aligned}
& \tau_{0} E(W)=\tau_{0}\left(P_{x \wedge(r+y)}-P_{x \wedge y}-P_{r \wedge(x-y)}\right) \\
& =\varphi(x \wedge(r+y)-x \wedge y-r \wedge(x-y))=0,
\end{aligned}
$$

where the last equality follows from checking the two cases where $x \geq r+y$ and $x<r+y$. Hence, $\tau$ is tracial, as claimed. This completes the proof.

For the index theory of Toeplitz operators on $H^{2}(\Gamma)$, where $\Gamma$ is an ordered group, the usual (classical) Fredholm index is not suitable, since all Fredholm Toeplitz operators are of index zero (for "most" ordered groups, see [16, Lemma 2.2]). Thus, the usual trace on $\mathcal{B}\left(H^{2}(\Gamma)\right)$, when restricted to the closed commutator ideal $\mathcal{K}(\Gamma)$ of the Toeplitz algebra $\mathcal{A}(\Gamma)$, does not give the right index theory. However, in the case that $\Gamma$ admits an order unit there is a trace on $\mathcal{K}(\Gamma)$ that gives a very satisfactory index theory, as we'll see in the following theorem.

This theorem has some overlap; in the special case that $\Gamma$ is an ordered subgroup of $\mathbf{R}$, with an earlier index theory result for Toeplitz operators on the Hardy space $H^{2}(\mathbf{R})$ with symbols that are almost periodic functions on $\mathbf{R}$, see [7. The latter result was one of the first theorems involving a real-valued index for Toeplitz operators. However, despite the overlap, this older result is in many respects different from our result, in that there is no reference to an invariant trace, and, in order to measure the index of a Toeplitz operator in the $H^{2}(\mathbf{R})$ setting, one has to represent the operator on a space different from $H^{2}(\mathbf{R})$ - there is no intrinsic formulation of the index theorem of the type shown here. Moreover, the construction of the representation used in [7] follows a very different line to the approach used in this paper (it is obtained in terms of the Murray-von Neumann group measure construction). A final point: The class of ordered groups is vastly more extensive than the class of ordered subgroups of $\mathbf{R}$, so our result is set in a more general framework than that discussed in 7 .

One should also note that our result is similar in some respects to the real-valued index theorem we obtained earlier in 16. However, there are again essential differences, both in the method of proof and in the actual result (the index theorem in 16] does not relate to a generalized Fredholm theory based on the commutator ideal, but on another, generally smaller, ideal; again, there is no intrinsic formulation of the index in [16]).

Recall now that if $\Gamma$ is an ordered group with dual group $G$, a nonzero topological index $\omega$ on $C(G)$ is distinguished if $\omega\left(\varepsilon_{x}\right) \geq 0$, for all $x \in \Gamma^{+}$. As we saw in Theorem 2.6, such a topological index $\omega$ exists if $\Gamma$ admits an order unit. In this case $\omega$ is unique up to multiplication by a positive constant.

Theorem 6.3. Let $\Gamma$ be an ordered group admitting an order unit and let $G$ denote its dual group. Then there exists a densely-defined lower semicontinuous trace $\mathrm{tr}$, 
unique up to multiplication by a positive constant, on the closed commutator ideal $\mathcal{K}(\Gamma)$ of $\mathcal{A}(\Gamma)$ that is invariant for the natural action of $G$ on $\mathcal{K}(\Gamma)$.

If $f \in C(G)$, then $T_{f}$ is a tr-Fredholm operator if, and only if, $f$ never vanishes on $G$. If $\omega$ is a distinguished topological index on $C(G)$, then there exists a positive constant $c$ such that

$$
\text { index }_{\operatorname{tr}}\left(T_{f}\right)=-c \omega(f) \quad(f \in \operatorname{Inv} C(G)) .
$$

(Of course, by suitable rescaling of $\omega$, we may suppose that $c=1$.)

Let $z$ be an order unit of $\Gamma$ and let $V$ be the essential isometry $T_{\varepsilon_{z}}$ in $\mathcal{A}(\Gamma)$. Then, by rescaling $\operatorname{tr}$ if necessary, the restriction of $\operatorname{tr}$ to $\mathcal{B}_{V}$ is the unique tracial state $\tau$ on $\mathcal{B}_{V}$ invariant for the natural action of $G$. In this case, if $\psi: \mathcal{A}(\Gamma) \rightarrow \mathcal{L}\left(H_{\mathcal{B}_{V}}^{2}\right)$ is the representation associated to $V$, then, for all $f \in C(G), T_{f}$ is a tr-Fredholm operator if, and only if, $\psi\left(T_{f}\right)$ is a $\operatorname{tr}_{\tau}$-Fredholm operator on $H_{\mathcal{B}_{V}}^{2}$, and then we have

$$
\operatorname{index}_{\operatorname{tr}}\left(T_{f}\right)=\operatorname{index}_{\tau} \psi\left(T_{f}\right) .
$$

Proof. Choose an order unit $z$ for $\Gamma$ and let $V=T_{\varepsilon_{z}}$ and $Q=Q_{V}$. Recall that $\mathcal{K}_{V}=\mathcal{K}(\Gamma)$ and that there is a unique surjective $*$-homomorphism $\pi: \mathcal{A}(\Gamma) \rightarrow C(G)$ for which $\pi\left(T_{f}\right)=f$, for all $f \in C(G)$ and that $\operatorname{ker}(\pi)=\mathcal{K}(\Gamma)$. Also, the Toeplitz map $T: C(G) \rightarrow \mathcal{A}(\Gamma), f \mapsto T_{f}$, is the $V$-related right inverse of $\pi$.

By Lemma6.2, $\mathcal{B}_{V}$ admits a unique tracial state $\tau$ invariant for the natural action $G$ on $\mathcal{B}_{V}$. Since $\operatorname{tr}_{\tau}$ is a densely-defined lower semicontinuous trace on $\mathcal{K}\left(H_{\mathcal{B}_{V}}^{2}\right)$, $\operatorname{tr}=\operatorname{tr}_{\tau} \circ \psi$ is a densely-defined lower semicontinuous trace on $\mathcal{K}(\Gamma)$. Clearly, the restriction $\operatorname{tr}_{\mathcal{B}_{V}}$ of $\operatorname{tr}$ to $\mathcal{B}_{V}$ is equal to $\tau$.

Now suppose $\operatorname{tr}^{\prime}$ is any lower semicontinuous trace on $\mathcal{K}(\Gamma)$. If $W \in \mathcal{K}(\Gamma)$, then $\operatorname{tr}^{\prime}\left(W^{*} W\right)=\lim _{n} \operatorname{tr}^{\prime}\left(W^{*} Q_{n} W\right)$, where $Q_{n}=1-V^{n} V^{* n}$, for all $n \geq 0$. Since $Q_{n+1}=Q_{n}+V^{n} Q V^{* n}$, for all $n$, we have

$$
\begin{gathered}
\operatorname{tr}^{\prime}\left(W^{*} W\right)=\sum_{n=0}^{\infty} \operatorname{tr}^{\prime}\left(W^{*}\left(Q_{n+1}-Q_{n}\right) W\right)=\sum_{n=0}^{\infty} \operatorname{tr}^{\prime}\left(W^{*} V^{n} Q V^{* n} W\right) \\
=\sum_{n=0}^{\infty} \operatorname{tr}^{\prime}\left(Q V^{* n} W W^{*} V^{n} Q\right)=\sum_{n=0}^{\infty} \tau^{\prime}\left(Q V^{* n} W W^{*} V^{n} Q\right),
\end{gathered}
$$

where $\tau^{\prime}$ is the restriction of $\operatorname{tr}^{\prime}$ to $\mathcal{B}_{V}{ }^{+}$. Also, $\operatorname{tr}^{\prime}$ is $G$-invariant if, and only if, $\tau^{\prime}$ is $G$-invariant for the natural action of $G$ on $\mathcal{B}_{V}$. Hence, in particular, tr is invariant for the natural action of $G$ on $\mathcal{K}(\Gamma)$. If now $\operatorname{tr}^{\prime}$ is a lower semicontinuous densely-defined trace on $\mathcal{K}(\Gamma)$, then $Q \in L^{1}\left(\operatorname{tr}^{\prime}\right)$ and so $\tau^{\prime} / \tau^{\prime}(Q)$ is a $G$-invariant tracial state on $\mathcal{B}_{V}$. Hence, $\tau^{\prime}=c \tau$, where $c=\tau^{\prime}(Q)>0$. It follows that, for all $W \in \mathcal{K}(\Gamma)$,

$$
\begin{gathered}
\operatorname{tr}^{\prime}\left(W^{*} W\right)=\sum_{n=0}^{\infty} \tau^{\prime}\left(Q V^{* n} W W^{*} V^{n} Q\right) \\
=\sum_{n=0}^{\infty} c \tau\left(Q V^{* n} W W^{*} V^{n} Q\right)=c \operatorname{tr}\left(W^{*} W\right),
\end{gathered}
$$

so that $\operatorname{tr}^{\prime}=c$ tr. 
Now let $\rho$ be the $*$-homomorphism from $C(G)$ to $C\left(\mathbf{T}, \mathcal{B}_{V}\right)=C^{*}\left(M_{\mathbf{z}}\right) \otimes \mathcal{B}_{V}$ given by Theorem 4.11. Since $\mathcal{K}(\Gamma)$ is essential as an ideal in $\mathcal{A}(\Gamma)$ [11, Theorem

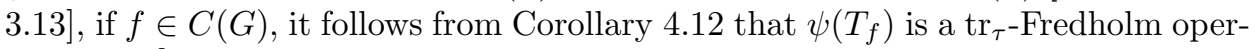
ator on $H_{\mathcal{B}_{V}}^{2}$ if, and only if, $f$ never vanishes on $G$ and in this case, index ${ }_{\tau} \psi\left(T_{f}\right)=$ $-\omega_{\tau} \rho(f)$. Hence, in this case, if $U$ is an operator in $\mathcal{A}(\Gamma)$ such that $1-T_{f} U$ and $1-U T_{f}$ belong to $L^{1}(\operatorname{tr})$, then

$$
\begin{gathered}
\text { index }_{\operatorname{tr}}\left(T_{f}\right)=\operatorname{tr}\left(T_{f} U-U T_{f}\right) \\
=\operatorname{tr}_{\tau}\left(\psi\left(T_{f}\right) \psi(U)-\psi(U) \psi\left(T_{f}\right)\right)=\operatorname{index}_{\tau} \psi\left(T_{f}\right) .
\end{gathered}
$$

The function

$$
\omega_{\tau} \rho: \operatorname{Inv} C(G) \rightarrow \mathbf{R}, \quad f \mapsto \omega_{\tau} \rho(f),
$$

is clearly a locally constant homomorphism and, by a remark preceding Theo$\operatorname{rem~3.3.~} \omega_{\tau} \rho\left(\varepsilon_{z}\right)=\omega_{\tau}(\mathbf{z} \otimes 1)=1$. If $x \in \Gamma$ and $x \geq 0$, then $1-T_{\varepsilon_{x}} T_{\varepsilon_{x}}^{*}$ is positive, since it is a projection, and therefore $1-\psi\left(T_{\varepsilon_{x}}\right) \psi\left(T_{\varepsilon_{x}}\right)^{*}$ is positive. Hence, $\omega_{\tau} \rho\left(\varepsilon_{x}\right)=-\operatorname{index}_{\tau} \psi\left(T_{\varepsilon_{x}}\right)=\operatorname{tr}_{\tau}\left(1-\psi\left(T_{\varepsilon_{x}}\right) \psi\left(T_{\varepsilon_{x}}\right)^{*}\right) \geq 0$. Therefore, $\omega_{\tau} \rho$ is a (nonzero) distinguished topological index on $C(G)$. Hence, if $\omega$ is any distinguished topological index on $C(G)$, there exists a positive number $c$ such that $c \omega=\omega_{\tau} \rho$. Therefore, if $f \in \operatorname{Inv} C(G)$, index $\operatorname{tr}\left(T_{f}\right)=\operatorname{index}_{\tau} \psi\left(T_{f}\right)=-\omega_{\tau} \rho(f)=-c \omega(f)$.

There is one fundamental point of difference between index theory for the case of an ordered subgroup $\Gamma$ of $\mathbf{R}$ and for non-Archimedean groups: If $\Gamma$ is an ordered subgroup of $\mathbf{R}$ and a Toeplitz operator $T_{f}$ has index zero, it is invertible. To see this, choose a densely-defined lower semicontinuous $G$-invariant trace $\operatorname{tr}$ on $\mathcal{K}(\Gamma)$ and a corresponding distinguished topological index $\omega$ on $C(G)$ such that index $_{\operatorname{tr}}\left(T_{f}\right)=-\omega(f)$, for all $f \in \operatorname{Inv} C(G)$. Since the function $x \mapsto \omega\left(\varepsilon_{x}\right)$ is a nonzero positive homomorphism from $\Gamma$ to $\mathbf{R}$, it is a positive constant multiple of the inclusion function $\Gamma \hookrightarrow \mathbf{R}$, so that for some positive number $c$ we have $\omega\left(\varepsilon_{x}\right)=c x$, for all $x \in \Gamma$. Now if $f \in \operatorname{Inv} C(G)$, then we can write it in the form $f=\varepsilon_{x} e^{h}$, for some continuous function $h$ on $G$ and a unique element $x$ of $\Gamma$, by Theorem 2.5. Then, if index $\operatorname{tr}\left(T_{f}\right)=0$, we have $c x=0$ and therefore $x=0$ so that $f=e^{h}$. However, it is shown in [13] that $T_{e^{h}}$ is necessarily invertible (the proof is easy).

On the other hand, if $\Gamma$ is a non-Archimedean group admitting an order unit and $x$ is any nonzero element of the largest proper ideal of $\Gamma$, then the preceding theorem implies that $T_{\varepsilon_{x}}$ is a tr-Fredholm operator of index zero, although it is noninvertible. This uses the fact that for any distinguished topological index $\omega$ on $C(G)$, the positive homomorphism

$$
\Gamma \rightarrow \mathbf{R}, \quad y \mapsto \omega\left(\varepsilon_{y}\right),
$$

vanishes on the largest proper ideal of $\Gamma$.

\section{INDEX THEORY FOR TOEPLITZ OPERATORS ON HARDY SPACES OF BOUNDED SYMMETRIC DOMAINS}

A bounded symmetric domain $D$ is said to be irreducible if it is not biholomorphically equivalent to the product $D_{1} \times D_{2}$ of two bounded symmetric domains $D_{1}$ and $D_{2}$. The irreducible domains are the most important ones. 
Theorem 7.1. Let $D$ be an irreducible tube-type symmetric open ball in $\mathbf{C}^{N}$, for some norm on $\mathbf{C}^{N}$. Let $V$ be the essential isometry $T_{\Delta}$ for the Toeplitz algebra $\mathcal{A}(D)$, where $\Delta$ is a Koecher norm for $D$.

Choose a tracial state $\tau$ for $\mathcal{B}_{V}$ (such states exist since $\mathcal{B}_{V}$ is a unital Type $I$ $C^{*}$-algebra). Let $\psi: \mathcal{A}(D) \rightarrow \mathcal{L}\left(H_{\mathcal{B}_{V}}^{2}\right)$ be the representation associated to $V$ and let tr be the lower semicontinuous densely-defined trace on the closed commutator ideal $\mathcal{K}(D)\left(=\mathcal{K}_{V}\right)$ of $\mathcal{A}(D)$ defined by setting $\operatorname{tr}(W)=\operatorname{tr}_{\tau} \psi(W)$, for all $W \in \mathcal{K}(D)^{+}$. The following conditions are equivalent, for a function $f \in C(S)$, where $S$ is the Shilov boundary of $D$ :

(1) $T_{f}$ is a tr-Fredholm operator;

(2) $\psi\left(T_{f}\right)$ is a $\operatorname{tr}_{\tau}$-Fredholm operator;

(3) $f$ never vanishes on $S$.

If these conditions hold, then

$$
\operatorname{index}_{\operatorname{tr}}\left(T_{f}\right)=\operatorname{index}_{\tau} \psi\left(T_{f}\right)=-\omega(f),
$$

where $\omega: \operatorname{Inv} C(S) \rightarrow \mathbf{R}$ is the distinguished topological index given by

$$
\omega(f)=\frac{1}{r} \int f^{-1} \sum_{j=1}^{N} z_{j} \frac{\partial f}{\partial z_{j}} \mathrm{~d} m,
$$

for all nonvanishing rational functions $f$ on $S$. Here $m$ is the Bott-Korányi measure on $S$ and $r$ is the rank of $D$.

Proof. Recall that there is a unique $*$-homomorphism $\pi: \mathcal{A}(D) \rightarrow C(S)$ such that $\pi\left(T_{f}\right)=f$, for all $f \in C(S)$ and that $\operatorname{ker}(\pi)=\mathcal{K}(D)$. It follows that $T_{f}$ is $\operatorname{tr}-$ Fredholm if, and only if, $f$ is invertible in $C(S)$; that is, $f$ never vanishes on $S$. Thus, Conditions (1) and (3) are equivalent.

It is also shown in 19] that $\mathcal{A}(D)$ acts irreducibly on $H^{2}(D)$, from which it follows that $\mathcal{K}(D)$ is essential as a closed ideal in $\mathcal{A}(D)$. It follows immediately, from Corollary 4.12, that Conditions (2) and (3) are equivalent.

Now we show the formula in Equation (10), and this will complete the proof of the theorem. Suppose then that $f$ is an element of $C(S)$ such that $T_{f}$ is trFredholm. Then there exists an operator $U$ in $\mathcal{A}(D)$ such that $1-T_{f} U$ and $1-U T_{f}$ belong to $L^{1}(\operatorname{tr})$. Hence, $1-\psi\left(T_{f}\right) \psi(U)$ and $1-\psi(U) \psi\left(T_{f}\right)$ belong to $L^{1}\left(\operatorname{tr}_{\tau}\right)$ and index $_{\operatorname{tr}}\left(T_{f}\right)=\operatorname{tr}\left(T_{f} U-U T_{f}\right)=\operatorname{tr}_{\tau}\left(\psi\left(T_{f}\right) \psi(U)-\psi(U) \psi\left(T_{f}\right)\right)=\operatorname{index}_{\tau} \psi\left(T_{f}\right)=$ $-\omega_{\tau} \rho(f)$, where we used Corollary 4.12 again to get the last equality.

Since $f \in \operatorname{Inv} C(S)$, we can write $f=\Delta^{n} e^{h}$, for some integer $n$ and element $h \in C(S)$ [1]. Hence, $\omega_{\tau} \rho(f)=\omega_{\tau}\left(\rho(\Delta)^{n} e^{\rho(h)}\right)=n \omega_{\tau} \rho(\Delta)=n \omega_{\tau}(\mathbf{z} \otimes 1)=n$. Also,

$$
\omega(\Delta)=\frac{1}{r} \int \bar{\Delta} \sum_{j=1}^{N} \frac{\partial \Delta}{\partial z_{j}} z_{j} \mathrm{~d} m=\frac{1}{r} \int \bar{\Delta} r \Delta \mathrm{d} m=1 .
$$

Therefore, $\omega(f)=\omega\left(\Delta^{n} e^{h}\right)=n \omega(\Delta)=n=\omega_{\tau} \rho(f)$. Thus, Equation (10) holds.

\section{REFERENCES}

[1] A.B. Badi, Index theory for generalized Toeplitz operators, Ph.D. thesis, National University of Ireland, Cork (2005).

[2] C.A. Berger, L.A. Coburn and A. Lebow, Representation and index theory for C*-algebras generated by commuting isometries, J. Funct. Anal. 27 (1978), 51-99. MR0467392 (57:7251) 
[3] C.A. Berger and L.A. Coburn, Wiener-Hopf operators on $U_{2}$, J. Integr. Equat. Oper. Th. 2 (1979), 139-173. MR543881 (81c:47031)

[4] H. Bohr, Über die Argumentvariation einer fastperiodischen Funktion, Danske vidensk Selskab. 10 (1930), 10.

[5] M. Breuer, Fredholm theories in von Neumann algebras I, Math. Ann. 178 (1968), 243-254. MR0234294 (38:2611)

[6] M. Breuer, Fredholm theories in von Neumann algebras II, Math. Ann. 180 (1969), 313-325. MR0264407 (41:9002)

[7] L.A. Coburn, R.G. Douglas, D. Schaeffer and I.M. Singer, C*-algebras of operators on a halfspace II. Index theory, Inst. Hautes Études Sci. Publ. Math. 40 (1971), 69-79. MR 0358418 $(50: 10884)$

[8] R.G. Douglas, Banach Algebra Techniques in Operator Theory, Academic Press, New YorkLondon (1972). MR 0361893 (50:14335)

[9] P.R. Halmos, A Hilbert Space Problem Book, Springer, New York-Heidelberg (1982). MR675952(84e:47001)

[10] E.C. Lance, Hilbert C*-Modules, Cambridge University Press, Cambridge (1995). MR.1325694 (96k:46100)

[11] G.J. Murphy, Ordered groups and Toeplitz algebras, J. Operator Theory 18 (1987), 303-326. MR915512 (89f:46132)

[12] G.J. Murphy, C*-algebras and Operator Theory, Academic Press, New York-London (1990). MR:1074574 (91m:46084)

[13] G.J. Murphy, Spectral and index theory for Toeplitz operators, Proc. Royal Irish Acad. 91 A (1991), 1-6. MR.1173153 (93k:47039)

[14] G.J. Murphy, Almost-invertible Toeplitz operators and K-Theory, J. Integr. Equat. Oper. Th. 15 (1992), 72-81. MR1134688 (93d:47056)

[15] G.J. Murphy, Toeplitz operators on generalised $H^{2}$ spaces, J. Integr. Equat. Oper. Th. 15 (1992), 825-852. MR.1177325 (93f:47026)

[16] G.J. Murphy, An index theorem for Toeplitz operators, J. Operator Theory 29 (1993), 97114. MR:1277967 (95h:47035)

[17] G.J. Murphy, Fredholm index and the trace, Proc. Royal Irish Acad. 94 A (1994), 161-166. MR1369029 (96m:47021)

[18] G.J. Murphy, C*-algebras generated by commuting isometries, Rocky Mountain J. Math. 26 (1996), 237-267. MR1386163 (97e:46074)

[19] G.J. Murphy, Toeplitz operators associated to unimodular algebras, J. Integr. Equat. Oper. Th. 46 (2003), 363-375. MR 1991785 (2004f:47033)

[20] G.J. Murphy, Topological and analytical indices in $\mathrm{C}^{*}$-algebras, preprint, National University of Ireland, Cork (2004), J. Funct. Anal. 234 (2006), 261-276. MR2216901 (2006m:46090)

[21] J. Phillips and I. Raeburn, An index theorem for Toeplitz operators with noncommutative symbol space, J. Funct. Anal. 120 (1994), 239-263. MR1266310 (95j:47035)

[22] S.C. Power, Commutator ideals and pseudodifferential $\mathrm{C}^{*}$-algebras, Quart. J. Math. Oxford 31 (1980), 467-489. MR596980 (82c:47033)

[23] W. Rudin, Fourier Analysis on Groups, Wiley, New York (1990). MR.1038803 (91b:43002)

[24] H. Upmeier, Toeplitz C*-algebras on bounded symmetric domains, Ann. Math. 119 (1984), 549-576. MR744863 (86a:47022)

[25] H. Upmeier, Fredholm indices for Toeplitz operators on bounded symmetric domains, Amer. J. Math. 110 (1988), 811-832. MR961496 (90f:47037)

[26] H. Upmeier, Toeplitz Operators and Index Theory in Several Complex Variables, Birkhaüser, Basel (1996). MR.1384981 (97f:47022)

[27] E. Van Kampen, On almost periodic functions of constant absolute value, J. London Math. Soc. 12 (1937), 3-6.

Department of Mathematics, National University of Ireland, Western Road, Cork, IRELAND 